\title{
Transport-limited water splitting at ion-selective interfaces during concentration
} polarization

\author{
Nielsen, Christoffer Peder; Bruus, Henrik
}

Published in:

Physical Review E

Publication date:

2014

Document Version

Publisher's PDF, also known as Version of record

Link back to DTU Orbit

Citation $(A P A)$ :

Nielsen, C. P., \& Bruus, H. (2014). Transport-limited water splitting at ion-selective interfaces during concentration polarization. Physical Review E, 89(4), 042405.

\section{General rights}

Copyright and moral rights for the publications made accessible in the public portal are retained by the authors and/or other copyright owners and it is a condition of accessing publications that users recognise and abide by the legal requirements associated with these rights.

- Users may download and print one copy of any publication from the public portal for the purpose of private study or research.

- You may not further distribute the material or use it for any profit-making activity or commercial gain

- You may freely distribute the URL identifying the publication in the public portal

If you believe that this document breaches copyright please contact us providing details, and we will remove access to the work immediately and investigate your claim. 


\title{
Transport-limited water splitting at ion-selective interfaces during concentration polarization
}

\author{
Christoffer P. Nielsen and Henrik Bruus \\ Department of Physics, Technical University of Denmark, DTU Physics Building 309, DK-2800 Kongens Lyngby, Denmark
}

(Received 11 December 2013; revised manuscript received 27 March 2014; published 15 April 2014)

\begin{abstract}
We present an analytical model of salt- and water-ion transport across an ion-selective interface based on an assumption of local equilibrium of the water-dissociation reaction. The model yields current-voltage characteristics and curves of water-ion current versus salt-ion current, which are in qualitative agreement with experimental results published in the literature. The analytical results are furthermore in agreement with direct numerical simulations. As part of the analysis, we find approximate solutions to the classical problem of pure salt transport across an ion-selective interface. These solutions provide closed-form expressions for the current-voltage characteristics, which include the overlimiting current due to the development of an extended space-charge region. Finally, we discuss how the addition of an acid or a base affects the transport properties of the system and thus provide predictions accessible to further experimental tests of the model.
\end{abstract}

DOI: 10.1103/PhysRevE.89.042405

PACS number(s): 68.05.-n, 47.57.jd, 82.39.Wj, 82.45.Mp

\section{INTRODUCTION}

Ion transport across an ion-selective interface, such as a nanochannel, an electrode, or an ion-selective membrane, has found numerous applications in, e.g., dialysis, desalination, battery and fuel cell technology, electrochemistry, and microfluidic systems [1-7]. A common feature of ion transport across ion-selective interfaces is the phenomenon known as concentration polarization, in which the ion concentration undergoes depletion next to the interface leading to a decrease in conductivity [1]. In the classical one-dimensional local electroneutrality (LEN) modeling of the problem, the conductivity goes to zero as the voltage drop over the system is increased, and the current approaches the so-called limiting current. Experimentally it has, however, been found that many concentration-polarized systems can sustain a significant overlimiting current $[1,8,9]$. A number of mechanisms have been suggested as explanation for this overlimiting current: these include the development of an extended space-charge region (ESC) [10-12], electro-osmotic instabilities (EOI) [13,14], water splitting $[15,16]$, current-induced membrane discharge (CIMD) [17], and surface conduction in microchannels [18]. Increasing amounts of evidence points to EOI as the primary mechanism in systems where advection is not suppressed by the geometry $[9,14]$. However, because of the experimental and theoretical difficulties associated with investigating concentration polarization, no unified picture describing the relative importance of mechanisms in different regimes has yet emerged. Concentration polarization is therefore still very much an open problem, warranting additional investigations into the underlying mechanisms.

In this paper, we investigate the effect of water splitting and an extended space-charge region on systems exhibiting concentration polarization. Apart from being relevant for classical concentration polarization in macroscopic systems, our investigation of water splitting is motivated by the recent studies which highlight the importance of reactions between hydronium and surface groups in microsystems [19-22].

Water splitting has long been investigated as a possible cause of overlimiting current in systems exhibiting concentration polarization $[15,23,24]$. In 1979, Kharkats predicted that besides adding to the total current in the system, a water-ion current is also able to increase, or exalt, the current of salt ions above the limiting current [16]. Since then, the effect and origin of the water-ion current has drawn considerable attention, and experiments have largely confirmed the fundamentals of Kharkats prediction [25-29]. It is reasonably well understood that the origin of the water-ion current is water dissociation taking place in a region close to the ion-selective interface. In many experiments, the magnitude of the water-ion current does, however, indicate a reaction rate much larger than what should be possible, considering only the bulk dissociation rates $[1,30]$. A number of models have been suggested to explain this remarkable feature. Some of these ascribe the increased reaction rate to catalytic interactions with membrane surface groups [25,31,32], while others use that the dissociation rate is increased in strong electric fields and employ a phenomenological function with one or more fitting parameters to describe this dependence $[26,30,33,34]$. In lack of conclusive evidence in support of either theory, the only thing that can be said with some confidence is that the actual reaction kinetics is probably exceedingly complicated.

In this work, we avoid the subject of the detailed reaction kinetics altogether by simply assuming that the dissociation rate is so large that the water-ion current is transport limited rather than reaction limited. Put in another way, we assume local equilibrium of the water ions everywhere in the system as done in Refs. [17,35]. Since the analysis given in this paper is based on this assumption, experiments supporting our conclusions would serve to corroborate the underlying assumption of local equilibrium of the water-dissociation reaction. In particular, the techniques allowing for individual measurements of salt current and water-ion current, such as titration-based methods $[26,27]$, are highly relevant, as many of our results and predictions depend explicitly on both these currents.

Even for systems where the water-equilibrium assumption is not justified, the presented analysis is valuable since it provides an upper bound to the currents which can be obtained (assuming that the equilibrium constant $K_{\mathrm{w}}$ remains fixed). Also, since the developed model employs a minimum of assumptions about the system, it is an excellent model to benchmark more detailed reaction models against. It has 


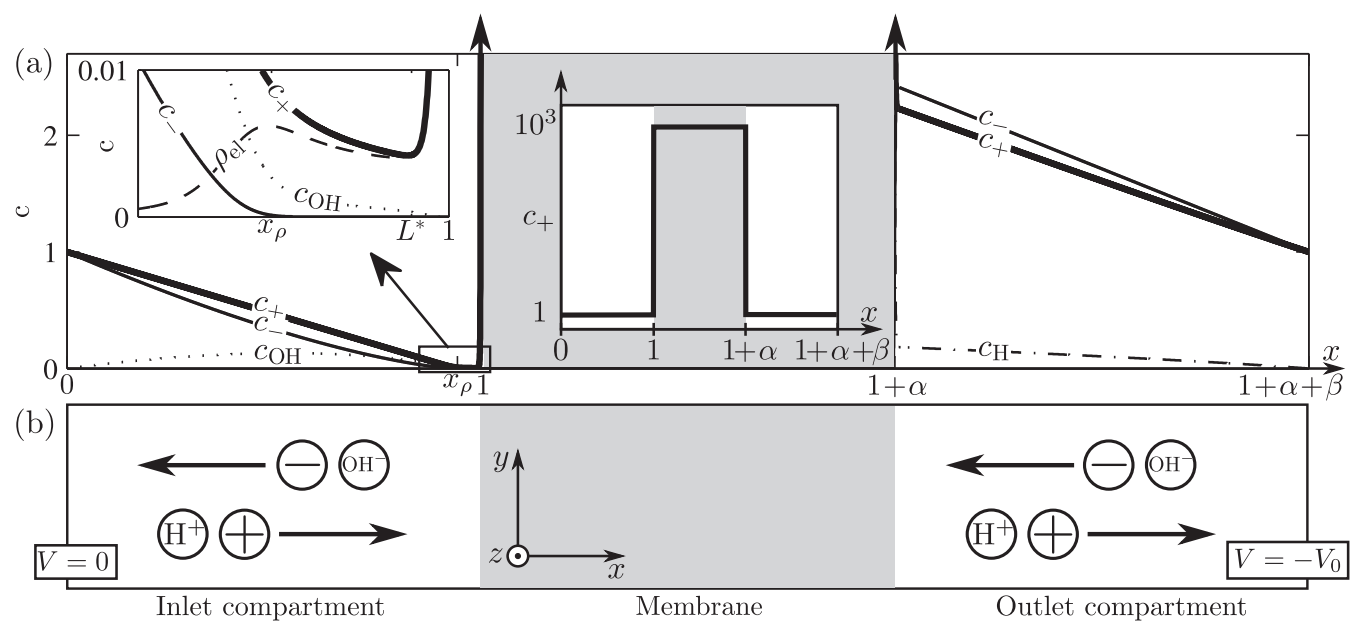

FIG. 1. (a) Normalized concentrations of salt ions $\left(c_{+}\right.$and $\left.c_{-}\right)$and water ions $\left(c_{\mathrm{OH}}\right.$ and $\left.c_{\mathrm{H}}\right)$ plotted versus normalized position $x$. The curves are obtained from a numerical simulation (see Sec. V). The top left inset is a zoom of the space-charge region (SCR) in front of the membrane. The SCR is composed of an extended space-charge region (ESC) from around $x_{\rho}$ to $L^{*}$, and a quasiequilibrium electric double layer (EDL) from $L^{*}$ to 1 . The center inset is a plot of the normalized cation concentration $c_{+}$showing the very high concentration inside the membrane $1<x<1+\alpha$ (gray). (b) Sketch of the studied system with salt ions $\left(+\right.$ and - ) and water ions $\left(\mathrm{H}^{+}\right.$and $\left.\mathrm{OH}^{-}\right)$. An inlet compartment $(0<x<1)$ and an outlet compartment $(1+\alpha<x<1+\alpha+\beta)$ separated by an ion-selective nanoporous membrane. To the left $(x=0)$ and right $(x=1+\alpha+\beta)$, the system is connected to reservoirs of well-defined salt concentration and $\mathrm{pH}$. The system is translationally invariant in the $y z$ plane parallel to the membrane.

for instance been a success criterion for reaction models that they are able to replicate the characteristic $S$ shape (increase-plateau-increase, see Sec. V B) of the experimental current-voltage curves [34]. However, such S-shaped currentvoltage curves are found even in our simple model, which suggests that they are a result of the transport properties of the system rather than the detailed reaction kinetics.

To simplify the treatment and bring forth the fundamental physics of water splitting, we study a system which is translationally invariant parallel to the ion-selective interface, and we use a one-dimensional (1D) model to describe this essentially one-dimensional system. By employing a 1D model, we disregard the possibility of spontaneous symmetry breaking, occurring at higher voltages in the form of electro-osmotic instabilities (EOI) [13,14], as this effect can only be described in a full three-dimensional (3D) model. For a number of systems where advection is suppressed by gels, microchannels, or porous structures, disregarding EOI is actually justified, and even when that is not the case our model provides a way to study the behavior before EOI sets in as well as the transition to EOI.

As a concrete realization of a system exhibiting concentration polarization, we investigate transport between two reservoirs across an ion-selective membrane. We have chosen this particular system for our study because the boundary conditions provided by the reservoirs are both simple and well defined. These features render the analysis conceptually simple, and for the water-splitting part of the problem, a reservoir is the simplest way of providing well-defined boundary conditions on the hydronium and hydroxide ions. Although the ideal reservoir boundary condition is a theoretical construct, it is possible to realize systems resembling the model system. An example of this is an electrodialysis cell as illustrated in Ref. [1]. Moreover, the simple solutions obtained from the studied system can be transformed into solutions for systems with more complicated boundary conditions. In Sec. VII we give examples of such transformations.

\section{MODEL SYSTEM}

The one-dimensional model system stretching along the $x$ axis is shown in Fig. 1. It consists of a central ionselective membrane of length $\alpha L$ connected to two well-mixed reservoirs, to the left and right, through two compartments of lengths $L$ and $\beta L$, respectively. The membrane is assumed to be much thicker than the local Debye length, so that the interior of the membrane can be treated as locally electroneutral. The reservoirs have well-defined $\mathrm{pH}$, and there is a potential difference $V_{0}$ between them. The left reservoir has salt concentration $c_{0}$ and the right reservoir has salt concentration $c_{\mathrm{R}} c_{0}$. The system is translationally invariant in the $y z$ plane parallel to the membrane. In Fig. 1(a) are shown typical normalized concentration distributions versus normalized position. These are obtained from the numerical simulations described in Sec. V. The top left inset shows the ion and charge concentrations in the space-charge region (SCR) near the membrane and two points $x_{\rho}$ and $L^{*}$ are defined for later use: $x_{\rho}$ denotes the position of the peak in space-charge density and $L^{*}$ denotes the beginning of the quasiequilibrium electric double layer (EDL). The part of the SCR lying outside the EDL, i.e., from around $x_{\rho}$ to $L^{*}$, we denote the extended space-charge region (ESC). Inside the membrane, the concentration of anions vanishes while the concentration of cations becomes very large $\left(\sim 10^{3}\right.$ times the reservoir concentration, depending on system parameters). In Fig. 1(b) is shown a sketch of the model system. The ions in the model are positive and negative salt ions with concentration $c_{+}$and $c_{-}$, respectively, as well as hydronium and hydroxide ions (water ions) with concentration $c_{\mathrm{H}}$ and $c_{\mathrm{OH}}$, respectively. 


\section{GOVERNING EQUATIONS}

We consider monovalent ions and normalize the ion concentrations by the reservoir salt concentration $c_{0}=c_{+}(0)=$ $c_{-}(0)$. The electrical potential $\phi$ is normalized by the thermal voltage $V_{\mathrm{T}}=k_{\mathrm{B}} T / e$ and the position by the length $L$. The cation current is normalized by the classical limiting current $J_{\lim }=2 D_{+} c_{0} / L$, the anion current is normalized by $2 D_{-} c_{0} / L$, while $J_{\mathrm{H}}$ and $J_{\mathrm{OH}}$ are both normalized by $2 D_{\mathrm{OH}} c_{0} / L$. The nondimensionalized ion currents are

$$
\begin{aligned}
2 J_{+} & =-\partial_{x} c_{+}-c_{+} \partial_{x} \phi, \\
2 J_{-} & =-\partial_{x} c_{-}+c_{-} \partial_{x} \phi, \\
2 J_{\mathrm{H}} & =-\delta \partial_{x} c_{\mathrm{H}}-\delta c_{\mathrm{H}} \partial_{x} \phi, \\
2 J_{\mathrm{OH}} & =-\partial_{x} c_{\mathrm{OH}}+c_{\mathrm{OH}} \partial_{x} \phi,
\end{aligned}
$$

where we have introduced the diffusivity ratio $\delta \equiv D_{\mathrm{H}} / D_{\mathrm{OH}}=$ 1.75. In the remainder of the paper, we are primarily concerned with nondimensional quantities. For the rare exceptions of dimensionful quantities, these will be indicated by a tilde.

In steady state the relevant Nernst-Planck equations for the salt ions are

$$
\partial_{x} J_{ \pm}=0 .
$$

Similar equations govern the motion of hydronium and hydroxide, with the addition of a reaction term $R$, which derives from the autoprotolytic reaction of water

$$
\begin{aligned}
& 0=-\partial_{x} J_{\mathrm{H}}+R, \\
& 0=-\partial_{x} J_{\mathrm{OH}}+R .
\end{aligned}
$$

Here, the reaction rates are identical since the reaction

$$
\mathrm{H}_{3} \mathrm{O}^{+}+\mathrm{OH}^{-} \rightleftharpoons 2 \mathrm{H}_{2} \mathrm{O}
$$

produces or consumes one unit of each species. Introducing the water-ion current $J_{\mathrm{w}} \equiv J_{\mathrm{H}}-J_{\mathrm{OH}}$ we obtain a single transport equation for the water ions

$$
\partial_{x} J_{\mathrm{w}}=0 .
$$

The fundamental assumption in this work is that the time scale of the autoprotolysis is much shorter than the transport time of hydronium and hydroxide. That is, we work in the limit of high Damköhler number, for which the hydronium and hydroxide concentrations are simply related via the equilibrium constant $K_{\mathrm{w}}=\tilde{c}_{\mathrm{OH}} \tilde{c}_{\mathrm{H}}$, which for dimensionless concentrations can be written as

$$
c_{\mathrm{OH}} c_{\mathrm{H}}=n^{2}, \quad \text { with } \quad n=\frac{\sqrt{K_{\mathrm{w}}}}{c_{0}} .
$$

The final governing equation is the Poisson equation

$$
\begin{aligned}
2 \bar{\lambda}_{\mathrm{D}}^{2} \partial_{x}^{2} \phi & =-c_{+}+c_{-}-c_{\mathrm{H}}+c_{\mathrm{OH}}, \\
\bar{\lambda}_{\mathrm{D}} & \equiv \frac{\lambda_{\mathrm{D}}}{L}=\frac{1}{L} \sqrt{\frac{\epsilon_{\mathrm{w}} V_{\mathrm{T}}}{2 e c_{0}}},
\end{aligned}
$$

where the nondimensionalized Debye length $\bar{\lambda}_{\mathrm{D}}$ has been introduced, with $e$ being the unit charge and $\epsilon_{\mathrm{w}}$ the permittivity of water. Since $\bar{\lambda}_{\mathrm{D}}^{2}$ is a small parameter, any small deviation from charge neutrality will greatly affect the potential in a manner which tends to restore charge neutrality. This observation is the basis of the often used local electroneutrality (LEN) assumption, where the bulk liquid is assumed electroneutral and the only deviation from electroneutrality is in the Debye layer.

The membrane is modeled as having a high density $N_{\mathrm{m}}$ of frozen negative charges (normalized by $c_{0}$ ), a porosity $\epsilon_{\mathrm{P}}$, a permittivity $\epsilon_{\mathrm{m}}$, and a tortuosity $\tau$. Inside the membrane, the currents and the Poisson equation are therefore modified as

$$
\begin{aligned}
2 J_{i} & =\frac{\epsilon_{\mathrm{P}}}{\tau}\left(-\partial_{x} c_{i} \pm c_{i} \partial_{x} \phi\right), \\
\frac{\epsilon_{\mathrm{m}}}{\epsilon_{\mathrm{W}}} 2 \bar{\lambda}_{\mathrm{D}}^{2} \partial_{x}^{2} \phi & =\epsilon_{\mathrm{P}}\left(-c_{+}+c_{-}-c_{\mathrm{H}}+c_{\mathrm{OH}}\right)+N_{\mathrm{m}} .
\end{aligned}
$$

Most ion-selective membranes have a complex structure [3638] making it difficult to properly determine the values of $N_{\mathrm{m}}$, $\epsilon_{\mathrm{P}}, \epsilon_{\mathrm{m}}$, and $\tau$. In this work we will, however, only consider cases with $N_{\mathrm{m}} \gg 1$, for which the potential drop over the membrane is negligible regardless of the membrane width $\alpha L$ and the value of the membrane parameters $N_{\mathrm{m}}, \epsilon_{\mathrm{P}}, \epsilon_{\mathrm{m}}$, and $\tau$.

The problem is closed by appropriate boundary conditions at either reservoir. At the left reservoir, the potential is set to zero and the normalized concentrations take the values $c_{ \pm}=1$, $c_{\mathrm{H}}=c_{\mathrm{OH}}=n$. At the right reservoir, the potential is set to $-V_{0}$ and the normalized concentrations take the values $c_{ \pm}=c_{\mathrm{R}}$, $c_{\mathrm{H}}=c_{\mathrm{OH}}=n$.

\section{ANALYTICAL TREATMENT}

In this section, we derive analytical expressions for the potential $\phi$ and concentration fields $c_{i}$ given as functions of the salt- and water-ion currents $J_{+}$and $J_{\mathrm{w}}$. As a result of the analysis, we find simple scaling laws for some of the characteristic features in the problem.

\section{A. Basic analysis}

For the simple system without water ions we know from Refs. [1,10,39] and numerical simulations that the left compartment is composed of three regions: a locally electroneutral diffusion layer, and a space-charge region (SCR), which can be subdivided into an extended space-charge region (ESC) and a quasiequilibrium electric double layer (EDL). This situation is sketched in Fig. 1(a).

Initially, we only consider the left compartment outside the EDL, i.e., the region extending from 0 to $L^{*}$ in the inset of Fig. 1(a). In the analysis we will assume that $L^{*}=1$, which as analyzed in the Supplemental Material, Sec. I A [40], is a good assumption for a wide range of parameter values. We introduce the effective water-ion density $c_{\mathrm{w}}$ and write

$$
\begin{aligned}
c_{\mathrm{w}} & \equiv \delta c_{\mathrm{H}}+c_{\mathrm{OH}}, \\
2 J_{\mathrm{w}} & =\partial_{x} c_{\mathrm{w}}-c_{\mathrm{w}} \partial_{x} \phi-2 \delta \partial_{x} c_{\mathrm{H}} \approx \partial_{x} c_{\mathrm{w}}-c_{\mathrm{w}} \partial_{x} \phi .
\end{aligned}
$$

We can discard the $2 \delta \partial_{x} c_{\mathrm{H}}$ term because the hydroxide concentration is very much larger than the hydronium concentration in the entire LEN region, and in the ESC region, where this may not be the case, diffusion plays a negligible role compared to electromigration.

We assume that the membrane is completely impenetrable to anions, so that $J_{-}=0$. The results can readily be 
generalized to the case of $J_{-} \neq 0$. Subtracting Eq. (1b) from Eq. (9b) we obtain

$$
\begin{aligned}
2 J_{\mathrm{w}} & =\partial_{x}\left(c_{\mathrm{w}}+c_{-}\right)-\left(c_{\mathrm{w}}+c_{-}\right) \partial_{x} \phi \\
& \approx \partial_{x} c_{+}-c_{+} \partial_{x} \phi+2 \bar{\lambda}_{\mathrm{D}}^{2} \partial_{x}^{3} \phi-2 \bar{\lambda}_{\mathrm{D}}^{2} \partial_{x} \phi \partial_{x}^{2} \phi,
\end{aligned}
$$

where Eq. (7a) with $(1+\delta) c_{\mathrm{H}} \approx 0$ has been used. This might introduce an error as we have just argued that $c_{\mathrm{W}} \gg c_{\mathrm{H}}$ does not necessarily hold in the ESC. The majority of the charge density in the ESC does, however, derive from the salt ions, so reasonable results may still be obtained with this approximation, as verified by our numerical simulations in Sec. V.

Adding (subtracting) Eq. (10b) to (from) Eq. (1a) we obtain

$$
\begin{aligned}
& J_{+}+J_{\mathrm{w}}=-c_{+} \partial_{x} \phi+\bar{\lambda}_{\mathrm{D}}^{2} \partial_{x}^{3} \phi-\bar{\lambda}_{\mathrm{D}}^{2} \partial_{x} \phi \partial_{x}^{2} \phi, \\
& J_{+}-J_{\mathrm{w}}=-\partial_{x} c_{+}-\bar{\lambda}_{\mathrm{D}}^{2} \partial_{x}^{3} \phi+\bar{\lambda}_{\mathrm{D}}^{2} \partial_{x} \phi \partial_{x}^{2} \phi .
\end{aligned}
$$

The second of these equations is easily integrated

$$
\left(J_{+}-J_{\mathrm{w}}\right) x-\kappa=-c_{+}-\bar{\lambda}_{\mathrm{D}}^{2} \partial_{x}^{2} \phi+\frac{\bar{\lambda}_{\mathrm{D}}^{2}}{2}\left(\partial_{x} \phi\right)^{2},
$$

where we have introduced the integration constant $\kappa=$ $c_{+}(0)+\left.\bar{\lambda}_{\mathrm{D}}^{2} \partial_{x}^{2} \phi\right|_{x=0}-\left.\frac{\bar{\lambda}_{\mathrm{D}}^{2}}{2}\left(\partial_{x} \phi\right)^{2}\right|_{x=0}$. For the studied system $\kappa$ is very close to unity since $c_{+}(0)=1$ and $-\left.\bar{\lambda}_{\mathrm{D}}^{2} \partial_{x}^{2} \phi\right|_{x=0}+$ $\left.\frac{\bar{\lambda}_{\mathrm{D}}^{2}}{2}\left(\partial_{x} \phi\right)^{2}\right|_{x=0} \ll 1$ for most relevant parameter values. In the following analysis, we keep $\kappa$ as an arbitrary constant. However, when plotting analytical results we have used the approximation $\kappa=1$. The validity of this approximation is studied in the Supplemental Material, Sec. I B [40].

Multiplying Eq. (12) by $\partial_{x} \phi$ and subtracting it from Eq. (11a), we obtain a single ordinary differential equation for the potential $\phi$ :

$$
J_{+}+J_{\mathrm{w}}-\left[\left(J_{+}-J_{\mathrm{w}}\right) x-\kappa\right] \partial_{x} \phi=\bar{\lambda}_{\mathrm{D}}^{2} \partial_{x}^{3} \phi-\frac{\bar{\lambda}_{\mathrm{D}}^{2}}{2}\left(\partial_{x} \phi\right)^{3} .
$$

This equation has previously been derived in various forms, for instance, in Refs. [10,39,41]. A common way of deriving solutions to this equation is to use the method of matched asymptotic expansions $[12,39,42,43]$. We will use a slightly simpler approach which omits the EDL, while still capturing the essential physics of the problem.

Let us consider the magnitude of the terms in Eq. (13) in each of the distinct regions. In the electroneutral diffusion layer, only the terms on the left of Eq. (13) matter since the entire right-hand side stem from the Poisson equation. In the ESC, the charge density can obviously not be neglected, and the terms on the right-hand side come into play. The right-hand side terms scale as $\bar{\lambda}_{\mathrm{D}}^{2} \partial_{x}^{3} \phi \sim \bar{\lambda}_{\mathrm{D}}^{2} \frac{\Delta \phi}{\Delta x^{3}}$ and $\bar{\lambda}_{\mathrm{D}}^{2}\left(\partial_{x} \phi\right)^{3} \sim \bar{\lambda}_{\mathrm{D}}^{2} \frac{\Delta \phi^{3}}{\Delta x^{3}}$, where $\Delta x$ and $\Delta \phi$ is the width of the ESC and the potential drop over the ESC, respectively. Because the conductivity in the ESC is small (few charge carriers), the potential drop over the ESC will be large. It follows that $\bar{\lambda}_{\mathrm{D}}^{2}\left(\partial_{x} \phi\right)^{3} \gg \bar{\lambda}_{\mathrm{D}}^{2} \partial_{x}^{3} \phi$, and as discussed in the Supplemental Material, Sec. I C [40], it is therefore reasonable to neglect the $\bar{\lambda}_{\mathrm{D}}^{2} \partial_{x}^{3} \phi$ term in Eq. (13). We then end up with a simple algebraic equation for the electric field, valid in the left compartment outside the EDL:

$$
1+\frac{\bar{\lambda}_{\mathrm{D}}^{2}}{2\left(J_{+}+J_{\mathrm{w}}\right)}\left(\partial_{x} \phi\right)^{3}=\left[\frac{J_{+}-J_{\mathrm{w}}}{J_{+}+J_{\mathrm{w}}} x-\frac{\kappa}{J_{+}+J_{\mathrm{w}}}\right] \partial_{x} \phi .
$$

Since $2 \bar{\lambda}_{\mathrm{D}}^{2} \partial_{x}^{3} \phi=-\partial_{x} \rho_{\mathrm{el}}$ the above assumption corresponds to assuming a quasiuniform distribution of the charge density. This method of simplifying the problem has previously been used by Urtenov et al. [41] and dubbed the assumption of quasiuniform charge density distribution. However, so far this assumption has only been used to simplify numerical calculations, and not to obtain analytical solutions.

To simplify the analysis, we introduce a scaled electric field $\hat{E}$ and a scaled position $\hat{x}$, defined by

$$
\hat{E} \equiv-B \partial_{x} \phi, \quad \text { with } \quad B \equiv\left[\frac{\bar{\lambda}_{\mathrm{D}}^{2}}{2\left(J_{+}+J_{\mathrm{w}}\right)}\right]^{1 / 3}
$$

and

$$
\hat{x} \equiv \frac{1}{B}\left[\frac{J_{+}-J_{\mathrm{w}}}{J_{+}+J_{\mathrm{w}}} x-\frac{\kappa}{J_{+}+J_{\mathrm{w}}}\right] .
$$

This enables us to recast Eq. (14) as

$$
-1+\hat{E}^{3}=\hat{x} \hat{E} .
$$

Before actually solving this equation, we can use it to derive some results characterizing the ESC. The scaled charge density $\hat{\rho}_{\mathrm{el}}=\partial_{\hat{x}} \hat{E}$ is found by implicit differentiation $3 \hat{E}^{2} \partial_{\hat{x}} \hat{E}=\hat{E}+$ $\hat{x} \partial_{\hat{x}} \hat{E}$, which results in

$$
\partial_{\hat{x}} \hat{E}=\frac{\hat{E}}{3 \hat{E}^{2}-\hat{x}} .
$$

Differentiating $\partial_{\hat{x}} \hat{E}$ again, it is found that the point of maximum charge density is at $\hat{x}=0$ and that

$$
\max \left(\hat{\rho}_{\mathrm{el}}\right)=\hat{\rho}_{\mathrm{el}}(0)=\frac{1}{3} .
$$

The simple form of this result is due to Eq. (17) being trivial for $\hat{x}=0$. The scaled charge density can be related to the unscaled charge density using

$$
\begin{aligned}
\rho_{\mathrm{el}} & =-2 \bar{\lambda}_{\mathrm{D}}^{2} \partial_{x}^{2} \phi=\frac{2 \bar{\lambda}_{\mathrm{D}}^{2}}{B} \partial_{x} \hat{x} \partial_{\hat{x}} \hat{E} \\
& =\left[32 \bar{\lambda}_{\mathrm{D}}^{2} \frac{\left(J_{+}-J_{\mathrm{w}}\right)^{3}}{J_{+}+J_{\mathrm{w}}}\right]^{1 / 3} \hat{\rho}_{\mathrm{el}} .
\end{aligned}
$$

From Fig. 1 it is seen that the point of maximum charge density marks the left edge of the ESC. Inserting $\hat{x}=0$ in Eq. (16) we find that the beginning of the ESC is at $x_{\rho}=\kappa /\left(J_{+}-J_{\mathrm{w}}\right)$, and therefore it has the width $1-x_{\rho}$. For the studied system $\kappa \approx 1$, so for $J_{+}-J_{\mathrm{w}}$ exceeding unity the extended space-charge region can make up a significant part of the channel. Inside the ESC, Eq. (17) yields $\hat{E} \sim \sqrt{\hat{x}}$ and the electric field scales as

$$
-\partial_{x} \phi=\frac{\hat{E}}{B} \sim \frac{\sqrt{\hat{x}}}{B}=\frac{\sqrt{2}}{\bar{\lambda}_{\mathrm{D}}} \sqrt{\left(J_{+}-J_{\mathrm{w}}\right) x-\kappa} .
$$

To leading order, these scalings are equivalent to others reported in the literature $[12,39,44]$. 


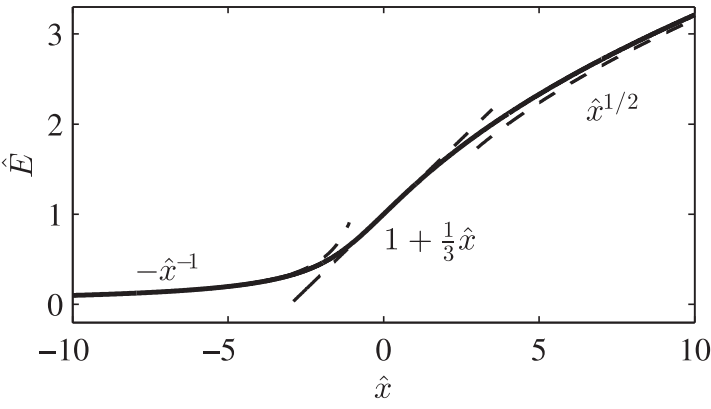

FIG. 2. Plot of scaled electric field $\hat{E}$ versus scaled position $\hat{x}$ (full line) from Eq. (23). The dashed lines show the limiting behavior for $\hat{x} \rightarrow-\infty, \hat{x} \rightarrow 0$, and $\hat{x} \rightarrow \infty$.

To proceed beyond this point, we write the general solution to Eq. (17):

$$
\begin{aligned}
\hat{E}= & -\frac{1}{2^{1 / 3}}\left(-1+\sqrt{1-\frac{4}{27} \hat{x}^{3}}\right)^{1 / 3} e^{i \omega} \\
& -\frac{2^{1 / 3}}{3} \hat{x}\left(-1+\sqrt{1-\frac{4}{27} \hat{x}^{3}}\right)^{-1 / 3} e^{-i \omega},
\end{aligned}
$$

where $\omega=0, \frac{2 \pi}{3}$, or $\frac{4 \pi}{3}$. We require that the solution is real and find

$$
\hat{E}=\left\{\begin{array}{lll}
\hat{E}_{-} & \text {for } \quad \hat{x} \leqslant 0, \\
\hat{E}_{+} & \text {for } \quad \hat{x} \geqslant 0,
\end{array}\right.
$$

which is continuous and differentiable at $\hat{x}=0$ and where

$$
\begin{aligned}
\hat{E}_{ \pm}= & \pm \frac{1}{2^{1 / 3}}\left( \pm 1 \mp \sqrt{1-\frac{4}{27} \hat{x}^{3}}\right)^{1 / 3} \\
& \pm \frac{2^{1 / 3}}{3} \hat{x}\left( \pm 1 \mp \sqrt{1-\frac{4}{27} \hat{x}^{3}}\right)^{-1 / 3} .
\end{aligned}
$$

In Fig. 2, the scaled electric field $\hat{E}$ is plotted for $-10<\hat{x}<$ 10 along with the asymptotic expressions.

It is noted that although this approach, like the method of matched asymptotic expansions [39,44,45], deals with different expressions inside and outside the ESC, the expressions used here are different branches of the same solution and as such they are matched by construction. This is a distinct advantage of the present approach, and it allows for an integration of the electric field to find the potential drop over the system.

We would like to relate the currents to the potential drop rather than the electric field. The task of integrating $\hat{E}$ is simplified considerably by using Eq. (18) to make a change of variable:

$$
\begin{aligned}
\hat{\phi} & =-\int \hat{E} d \hat{x}=-\int \hat{E} \frac{1}{\partial_{\hat{x}} \hat{E}} d \hat{E}=-\int 2 \hat{E}^{2}+\frac{1}{\hat{E}} d \hat{E} \\
& =-\frac{2}{3}\left[\hat{E}^{3}-\hat{E}^{3}\left(\hat{x}_{0}\right)\right]-\ln \left(\frac{\hat{E}}{\hat{E}\left(\hat{x}_{0}\right)}\right),
\end{aligned}
$$

where $\hat{x}_{0} \equiv-\kappa /\left[B\left(J_{+}+J_{\mathrm{w}}\right)\right]$ [Eq. (16) with $\left.x=0\right]$. Equivalently, we define $\hat{x}_{1} \equiv\left(J_{+}-J_{\mathrm{w}}-\kappa\right) /\left[B\left(J_{+}+J_{\mathrm{w}}\right)\right]$ [Eq. (16) with $x=1]$.
The unscaled potential $\phi$ is related to the scaled potential $\hat{\phi}$ as

$$
\phi=\int \partial_{x} \phi d x=-\frac{1}{B} \frac{1}{\partial_{x} \hat{x}} \int \hat{E} d \hat{x}=j \hat{\phi},
$$

where $j \equiv\left(J_{+}+J_{\mathrm{w}}\right) /\left(J_{+}-J_{\mathrm{w}}\right)$ has been introduced for convenience. At the inlet $\hat{E}$ is small so we can make the approximations $\hat{E}^{3}\left(\hat{x}_{0}\right) \approx 0$ and $\hat{E}\left(\hat{x}_{0}\right) \approx-\frac{1}{\hat{x}_{0}}$ and find the simpler expression

$$
\phi \approx-\frac{2}{3} j \hat{E}^{3}-j \ln \left(-\hat{E} \hat{x}_{0}\right) .
$$

The cation concentration is obtained from Eq. (12),

$$
c_{+}=\frac{\bar{\lambda}_{\mathrm{D}}^{2}}{B^{2}}\left[\frac{1}{2 \hat{E}}+\frac{1}{j} \partial_{\hat{x}} \hat{E}\right],
$$

and since the anions are Boltzmann distributed,

$$
c_{-}=e^{\phi}=e^{j \hat{\phi}} .
$$

To make the further calculation internally consistent, we again use $(1+\delta) c_{\mathrm{H}} \approx 0$, and find from the Poisson equation that

$$
c_{\mathrm{w}}=c_{+}-c_{-}-\rho_{\mathrm{el}}=\frac{\bar{\lambda}_{\mathrm{D}}^{2}}{B^{2}}\left[\frac{1}{2 \hat{E}}-\frac{1}{j} \partial_{\hat{x}} \hat{E}\right]-e^{j \hat{\phi}} .
$$

In conclusion, our model gives analytical expressions for all the relevant fields $\phi, c_{ \pm}$, and $c_{\mathrm{w}}$ as a function of the position $x$ and the salt- and water-ion currents $J_{+}$and $J_{\mathrm{w}}$. This part of the analysis is completely general and does not rely on the specific type of ion-selective interface; the nature of the ion-selective interface is only important for the behavior inside the EDL.

In the Supplemental Material [40], we provide an analysis of the limitations of the approximations employed in this section. We find that the derived expressions are valid when $\bar{\lambda}_{\mathrm{D}}^{2} J_{+}^{2} \ll 1$ and $\bar{\lambda}_{\mathrm{D}} \ll 1$. In the overlimiting regime, it also has to be the case that $\bar{\lambda}_{\mathrm{D}}^{2 / 3} J_{+}^{-1 / 3} \ll 1$. We also find corrections to the theory which can extend its validity beyond these limits. However, for typical system parameters these corrections are minute, and for that reason we have chosen just to state them in the Supplemental Material [40].

\section{B. Case without water-ion current}

Initially, we consider the simple case of zero water-ion current $J_{\mathrm{w}}=0$. In this limit, the problem only depends on the parameter $\bar{\lambda}_{\mathrm{D}}$ and the potential is given by Eq. (25). Since this result gives a closed-form expression for the potential, valid at both underlimiting and overlimiting currents, we consider it to be an extension of earlier asymptotic expressions, valid only in the overlimiting regime, given in Refs. [11,12].

To find the approximate dependence on $\bar{\lambda}_{\mathrm{D}}$ we consider the limit $\hat{E}^{3}\left(\hat{x}_{1}\right) \gg 1$, for which $\hat{E}\left(\hat{x}_{1}\right)$ is given by $\hat{E}\left(\hat{x}_{1}\right) \approx \sqrt{\hat{x}_{1}}$ and the potential at $x=1$ becomes

$$
\begin{aligned}
\phi(1) & \approx-\frac{2}{3} \hat{x}_{1}^{3 / 2}-\ln \left(-\sqrt{\hat{x}_{1}} \hat{x}_{0}\right) \\
& =-\frac{2}{3}\left[\frac{2\left(J_{+}-\kappa\right)^{3}}{\bar{\lambda}_{\mathrm{D}}^{2} J_{+}^{2}}\right]^{1 / 2}-\frac{1}{2} \ln \left[\frac{2\left(J_{+}-\kappa\right)}{\bar{\lambda}_{\mathrm{D}}^{2} J_{+}^{2}}\right] \\
& \approx-\frac{2 \sqrt{2}}{3} \frac{\left(J_{+}-\kappa\right)^{3 / 2}}{\bar{\lambda}_{\mathrm{D}} J_{+}}+\ln \left(\bar{\lambda}_{\mathrm{D}}\right) .
\end{aligned}
$$




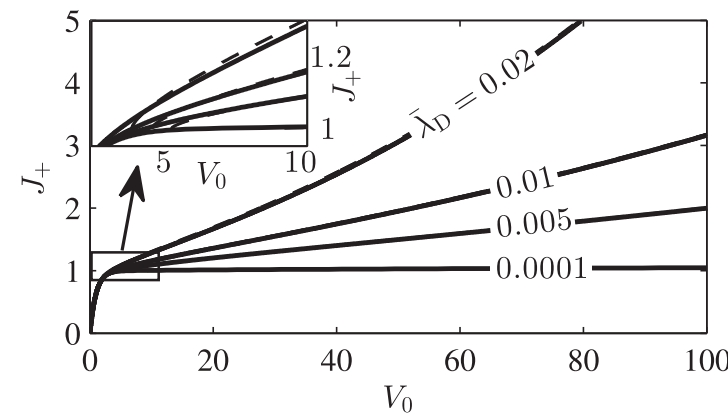

FIG. 3. Salt current $J_{+}$plotted versus voltage $V_{0}$ in the case of vanishing water-ion current $J_{\mathrm{w}}$ and for $\kappa=1$. Full line is the analytical expression (25), and the dashed line is the asymptotic expression (27). Only near the limiting current $J_{+} \gtrsim 1$ do the two cases deviate appreciably (see the inset).

The first term on the right-hand side dominates, so for a given overlimiting current, the potential drop will roughly scale with $\bar{\lambda}_{\mathrm{D}}^{-1}$. This agrees well with the intuitive picture, that the more strictly electroneutrality is enforced, the greater is the potential drop required to create the ESC and drive a current. In Fig. 3, the current is plotted versus the voltage difference for varying $\bar{\lambda}_{\mathrm{D}}$ and $\kappa=1$. The full analytical solution is shown with a full line and the asymptotic solution is shown with a dashed line (only for $J_{+}>1$ ).

\section{Influence of water ions}

To find a relation between $J_{+}$and $J_{\mathrm{w}}$, when water ions are taken into account, we need another constraint on one of the fields. It is, however, not apparent which constraint we should use or, for that matter, that a simple and physically justified constraint even exists. In the numerical simulations, as we shall later see, the value of $J_{\mathrm{w}}$ is determined self-consistently by simply requiring continuity of the fields through the membrane. The analytical model does, however, break down in the EDL, so this method of constraining $J_{\mathrm{w}}$ can not be employed here.

Instead, we use a boundary condition which is not entirely rigorous, but does have the appeal of being very simple. Let us consider Eq. (9b) in the ESC where diffusion is small compared to electromigration:

$$
2 J_{\mathrm{w}} \approx-c_{\mathrm{w}} \partial_{x} \phi
$$

There is a positive charge density in the ESC so the electric field increases for increasing $x$. Because $J_{\mathrm{w}}$ is divergence free, this in turn means that $c_{\mathrm{w}}$ must decrease for increasing $x$. However, $c_{\mathrm{w}}$ has a minimum value $\min \left(c_{\mathrm{w}}\right)=2 \sqrt{\delta} n$ because of the relation Eq. (6), so at $x=1$ we must always have $J_{\mathrm{w}} \geqslant$ $-\sqrt{\delta} n \partial_{x} \phi$. For all but the lowest currents (whose contributions are negligible), it seems that this is indeed the constraint which creates the water-ion current, i.e., we determine the water-ion current from

$$
\left.\partial_{x} \phi\right|_{x=1}=-\frac{J_{\mathrm{w}}}{\sqrt{\delta} n} .
$$

By inserting this in Eq. (14) and solving for $J_{+}$, we find a relation between $J_{+}$and $J_{\mathrm{w}}$ :

$$
J_{+}=J_{\mathrm{w}} \frac{\kappa+J_{\mathrm{w}}+\frac{\bar{\lambda}_{\mathrm{D}}^{2}}{2 \delta n^{2}} J_{\mathrm{w}}^{2}}{\sqrt{\delta} n+J_{\mathrm{w}}} .
$$

Using this relation together with Eq. (25), the current-voltage characteristic for the system can be evaluated for any set of parameters. We note that this boundary condition is the only place where the equilibrium constant enters in the analysis, so a more general treatment allowing the equilibrium constant to vary can be implemented by an appropriate modification of $n$ in Eq. (30).

It is instructive to consider some limiting cases. For overlimiting currents, where $J_{\mathrm{w}} \gg \sqrt{\delta} n$, Eq. (30) yields a simple expression for $J_{\mathrm{w}}$ in terms of $J_{+}$:

$$
J_{\mathrm{w}} \approx \frac{\delta n^{2}}{\bar{\lambda}_{\mathrm{D}}^{2}}\left(-1+\sqrt{1+\frac{2 \bar{\lambda}_{\mathrm{D}}^{2}}{\delta n^{2}}\left(J_{+}-\kappa\right)}\right) .
$$

Expanding this in the two limits $\frac{2 \bar{\lambda}_{\mathrm{D}}^{2}}{\delta n^{2}}\left(J_{+}-\kappa\right) \sim \frac{\bar{\lambda}_{\mathrm{D}}^{2}}{n^{2}} \ll 1$ and $\frac{2 \bar{\lambda}_{\mathrm{D}}^{2}}{\delta n^{2}}\left(J_{+}-\kappa\right) \sim \frac{\bar{\lambda}_{\mathrm{D}}^{2}}{n^{2}} \gg 1$, we find

$$
J_{\mathrm{w}} \approx \begin{cases}J_{+}-\kappa & \text { for } \quad \frac{\bar{\lambda}_{\mathrm{D}}^{2}}{n^{2}} \ll 1, \\ \frac{\sqrt{2 \delta} n}{\bar{\lambda}_{\mathrm{D}}} \sqrt{J_{+}-\kappa} & \text { for } \quad \frac{\bar{\lambda}_{\mathrm{D}}^{2}}{n^{2}} \gg 1 .\end{cases}
$$

The first of these limits we denote the Kharkats limit since he studied exactly the situation $J_{+}=1+J_{\mathrm{w}}$ where the overlimiting current is only due to screening by water ions [16]. The potential drop over the system is given by Eq. (25c), and using that $\hat{E}\left(\hat{x}_{1}\right)=-\left.B \partial_{x} \phi\right|_{x=1}=B \frac{J_{\mathrm{w}}}{\sqrt{\delta} n}$ we find

$$
\begin{aligned}
\phi(1) & \approx-\frac{2}{3} j\left(B \frac{J_{\mathrm{w}}}{\sqrt{\delta} n}\right)^{3}-j \ln \left(-B \frac{J_{\mathrm{w}}}{\sqrt{\delta} n} \hat{x}_{0}\right) \\
& =-\frac{\bar{\lambda}_{\mathrm{D}}^{2}}{3\left(J_{+}-J_{\mathrm{w}}\right)}\left(\frac{J_{\mathrm{w}}}{\sqrt{\delta} n}\right)^{3}-j \ln \left(\frac{J_{\mathrm{w}}}{\sqrt{\delta} n} \frac{\kappa}{J_{+}+J_{\mathrm{w}}}\right) .
\end{aligned}
$$

An interesting feature of this result is that even in the Kharkats limit $\bar{\lambda}_{\mathrm{D}}^{2} / n^{2} \ll 1$, where the entire overlimiting current is due to water-ion screening, the potential depends on $\bar{\lambda}_{\mathrm{D}}$.

In the case of overlimiting current, the potential drop is determined by inserting Eq. (31) in Eq. (33). In the Kharkats limit $\bar{\lambda}_{\mathrm{D}}^{2} / n^{2} \ll 1$ given in Eq. (32), we obtain

$$
\begin{aligned}
\phi(1) \approx & -\frac{\bar{\lambda}_{\mathrm{D}}^{2}}{3}\left(\frac{J_{+}-\kappa}{\sqrt{\delta} n}\right)^{3} \\
& -\left(\frac{2}{\kappa} J_{+}-1\right) \ln \left(\frac{J_{+}-\kappa}{\sqrt{\delta} n} \frac{1}{2 J_{+}-\kappa}\right),
\end{aligned}
$$

while in the opposite limit $\bar{\lambda}_{\mathrm{D}}^{2} / n^{2} \gg 1$ we find

$$
\phi(1) \approx-\frac{2 \sqrt{2}}{3} \frac{\left(J_{+}-\kappa\right)^{3 / 2}}{\bar{\lambda}_{\mathrm{D}} J_{+}}+\ln \left(\bar{\lambda}_{\mathrm{D}}\right) .
$$

A remarkable conclusion can immediately be drawn from these expressions. In the limit $\bar{\lambda}_{\mathrm{D}}^{2} / n^{2} \ll 1$, the potential drop for a given normalized current $J_{+}$is seen to increase with $\bar{\lambda}_{\mathrm{D}}$. This is opposite to the conclusion in the $J_{\mathrm{w}}=0$ analysis, and it can be viewed as a result of the coupling between $J_{\mathrm{w}}$ and 


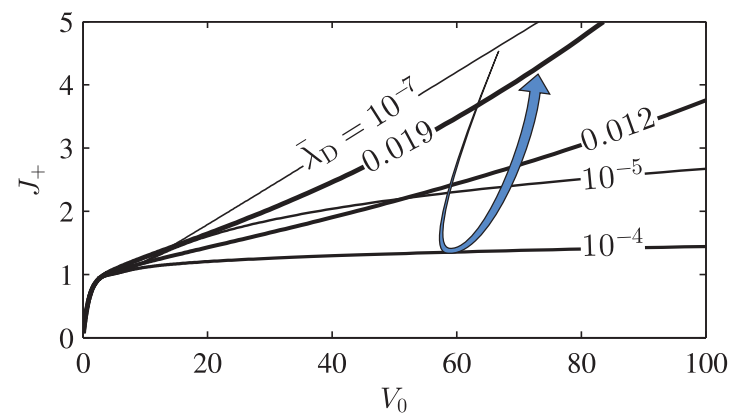

FIG. 4. (Color online) Salt current $J_{+}$from Eqs. (25) and (30) plotted versus voltage $V_{0}$ for $n=10^{-4}, \kappa=1$, and $\bar{\lambda}_{\mathrm{D}}$ varying from $10^{-7}$ (thin) to 0.019 (thick). The curved arrow indicates the nonmonotonous dependence on $\bar{\lambda}_{\mathrm{D}}$.

$\partial_{x} \phi$, which is brought about by the boundary condition (29). We also see that the potential drop scales inversely with $n$ as expected.

In the other limit $\bar{\lambda}_{\mathrm{D}}^{2} / n^{2} \gg 1$, we recover the $\bar{\lambda}_{\mathrm{D}}^{-1}$ scaling from the $J_{\mathrm{w}}=0$ analysis as well as the $\phi$ expression (27). The potential drop over the system will thus have a nonmonotonous dependence on $\bar{\lambda}_{D}$. This behavior is seen in Fig. 4, where the salt current $J_{+}$is plotted versus voltage for fixed $n$ and varying $\bar{\lambda}_{D}$. It is seen that for some parameter values, e.g., $\bar{\lambda}_{D}=10^{-5}$ we obtain the characteristic $S$-shaped current-voltage curve found in experiments $[8,26,46,47]$. This indicates, at least on a qualitative level, that the developed model captures the relevant physics of the problem.

\section{Concentration fields}

The concentration fields found in our analysis exhibit a very rich structure, and it is generally difficult to describe their behavior in simple terms.

In Fig. 5, the concentration fields are shown for a given set of parameters. Outside the ESC, the fields behave as in the simple LEN theory, with $c_{+}$decreasing linearly with $x, c_{-}$ scaling as $\left(c_{+}\right)^{j}$, and $c_{\mathrm{w}}$ given by the difference $c_{+}-c_{-}$:

$$
\begin{aligned}
& c_{+} \approx \kappa-\left(J_{+}-J_{\mathrm{w}}\right) x, \\
& c_{-} \approx\left[\kappa-\left(J_{+}-J_{\mathrm{w}}\right) x\right]^{j}, \\
& c_{\mathrm{w}} \approx \kappa-\left(J_{+}-J_{\mathrm{w}}\right) x-\left[\kappa-\left(J_{+}-J_{\mathrm{w}}\right) x\right]^{j} .
\end{aligned}
$$

Since $J_{\mathrm{w}}$ is determined via Eq. (30), these seemingly simple relations do in fact have a quite complicated dependence on all of the parameters $J_{+}, \bar{\lambda}_{\mathrm{D}}$, and $n$. It is seen that the expressions break down inside the extended space-charge region $x>x_{\rho}=$ $\kappa /\left(J_{+}-J_{\mathrm{w}}\right)$.

In the ESC, the existence of a nonzero charge density complicates matters further. The charge density has a peak at the beginning of the ESC,

$$
\rho_{\mathrm{el}}\left(x_{\rho}\right)=\frac{2}{3} 2^{2 / 3} \bar{\lambda}_{\mathrm{D}}^{2 / 3}\left(J_{+}-J_{\mathrm{w}}\right)\left(J_{+}+J_{\mathrm{w}}\right)^{-1 / 3},
$$

and in the ESC it decays as

$$
\rho_{\mathrm{el}} \approx 2^{1 / 2} \bar{\lambda}_{\mathrm{D}}\left(J_{+}-J_{\mathrm{w}}\right)\left[\left(J_{+}-J_{\mathrm{w}}\right) x-\kappa\right]^{-1 / 2} .
$$

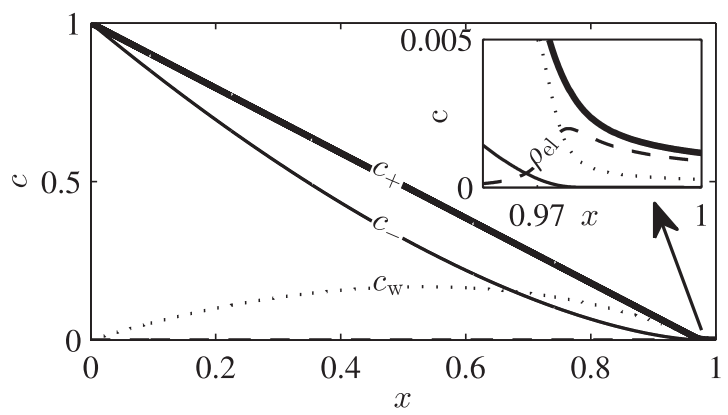

FIG. 5. The analytical expression [Eq. (26)] for the concentration fields $c_{+}, c_{-}, c_{\mathrm{w}}$, and $\rho_{\mathrm{el}}$ plotted versus position $x$ for $n=10^{-4}$, $\bar{\lambda}_{\mathrm{D}}=10^{-4}, \kappa=1$, and $J_{\mathrm{w}}=0.3$. The corresponding salt current is found from Eq. (30) to be $J_{+}=1.325$. The inset shows the behavior in the ESC close to the membrane.

In the limit $\bar{\lambda}_{\mathrm{D}}^{2} / n^{2} \gg 1$, where the influence of water ions is negligible, the expressions simplify as

$$
\begin{aligned}
\rho_{\mathrm{el}}\left(x_{\rho}\right) & \approx \frac{2}{3} 2^{2 / 3} \bar{\lambda}_{\mathrm{D}}^{2 / 3} J_{+}^{2 / 3}, \\
\rho_{\mathrm{el}} & \approx 2^{1 / 2} \bar{\lambda}_{\mathrm{D}} J_{+}\left(J_{+} x-\kappa\right)^{-1 / 2} .
\end{aligned}
$$

In this case, both the peak charge density and the charge density inside the ESC increase with $J_{+}$.

In the Kharkats limit $\bar{\lambda}_{\mathrm{D}}^{2} / n^{2} \ll 1$, where $J_{+} \approx \kappa+J_{\mathrm{w}}$, the charge density at $x_{\rho}$ simplifies as

$$
\rho_{\mathrm{el}}\left(x_{\rho}\right) \approx \frac{2}{3} 2^{2 / 3} \bar{\lambda}_{\mathrm{D}}^{2 / 3} \kappa\left(2 J_{+}-\kappa\right)^{-1 / 3} .
$$

Here, the peak charge density surprisingly decreases with increasing $J_{+}$. Also, in this limit the ESC will be very small since $x_{\rho}=\kappa /\left(J_{+}-J_{\mathrm{w}}\right) \approx 1$. The reduction in width and magnitude of the ESC will act to suppress EOI in the $\bar{\lambda}_{\mathrm{D}}^{2} / n^{2} \ll 1$ limit. This is similar to the effect of currentinduced membrane discharge as described in Ref. [17]. In the literature it has been reported that EOI sets in around $V_{0}=20$ [48]. As seen from Fig. 4 and the results in Sec. V B, water splitting sets in at a lower voltage, which leads us to believe that a suppression of EOI will in fact occur in this limit.

\section{E. Total potential drop}

The developed analytical model gives a general description, valid for any ion-selective interface, of the inlet compartment outside the EDL. To enable comparison with the numerical simulations of a membrane system, a simple model for the potential drop over the remainder of the system is developed.

Inside the membrane there is a very large density $N_{\mathrm{m}}$ of immobile negative charges. To screen these charges, an equally large density of positive ions accumulates. It follows that the conductivity in the membrane is very large, so that the potential drop over the membrane is negligible compared to the other potential drops in the system. While the potential drop inside the membrane can safely be neglected, the potential drops $\Delta \phi_{\mathrm{m} 1}$ and $\Delta \phi_{\mathrm{m} 2}$ over the two membrane interfaces are in general non-negligible. To determine them, we use the assumption of quasiequilibrium to relate the concentrations just outside the membrane to the concentrations inside the membrane via a Boltzmann factor. Charge neutrality in the 
membrane then gives

$$
\begin{aligned}
0 & =-N_{\mathrm{m}}+\epsilon_{\mathrm{P}}\left(c_{+}-c_{-}+c_{\mathrm{H}}-c_{\mathrm{OH}}\right) \\
& \approx-N_{\mathrm{m}}+\epsilon_{\mathrm{P}}\left[c_{+}(1)+c_{\mathrm{H}}(1)\right] e^{-\Delta \phi_{\mathrm{m} 1}},
\end{aligned}
$$

where we used that the concentration of anions in the membrane is negligible. The same argument applies to both membrane interfaces, so the total potential change across the membrane is

$$
\Delta \phi_{\mathrm{m}}=\ln \left(\frac{c_{+}(1)+c_{\mathrm{H}}(1)}{c_{+}(1+\alpha)+c_{\mathrm{H}}(1+\alpha)}\right) .
$$

In the outlet channel, local charge neutrality is an excellent approximation and the water-ion current is totally dominated by hydronium. From the transport equations [Eqs. (1) and (2)], we therefore find

$$
\begin{aligned}
c_{+}+c_{\mathrm{H}}=c_{-} & =c_{\mathrm{R}} e^{\phi+V_{0}}, \\
c_{\mathrm{R}}+\left(J_{+}+\frac{J_{\mathrm{w}}}{\delta}\right)(1+\alpha+\beta-x) & =c_{\mathrm{R}} e^{\phi+V_{0}} .
\end{aligned}
$$

Here, we used that $1+\alpha<x<1+\alpha+\beta$ in the outlet channel and $\phi(1+\alpha+\beta)=-V_{0}$. In conclusion, the total potential drop across the entire system is

$$
\begin{aligned}
V_{0}= & -[\phi(1)-\phi(0)]-\Delta \phi_{\mathrm{m}}-[\phi(1+\alpha+\beta)-\phi(1+\alpha)] \\
= & -\phi(1)-\ln \left[c_{+}(1)+c_{\mathrm{H}}(1)\right] \\
& +2 \ln \left[c_{\mathrm{R}}+\beta\left(J_{+}+\frac{J_{\mathrm{w}}}{\delta}\right)\right],
\end{aligned}
$$

where $\phi(1)$ is given in Eq. (25), $c_{+}$and $c_{\mathrm{H}}$ are given in Eq. (26), and the relation between the currents is given in Eq. (30). It is seen that in the limit $\beta=0$ and $c_{\mathrm{R}}=1$, the potential drop is just the change in electrochemical potential of the positive ions from inlet reservoir to membrane.

\section{NUMERICAL SIMULATIONS}

\section{A. Numerical implementation}

The numerical simulations are carried out in the commercially available finite element software COMSOL MULTIPHYSICS (ver. 4.3a). Following Gregersen et al. [49], the equations (1), (2), (5), (6), (7a), and (8) are rewritten in weak form and implemented in the mathematics module of COMSOL. In the numerical simulations and in the comparisons with theory we have used $\alpha=\beta=1$ and $c_{\mathrm{R}}=1$. This choice only gives a small loss of generality since the results are weakly sensitive to these values, as seen from Eq. (42). We use the following boundary conditions: $c_{ \pm}(0)=1, c_{\mathrm{H}}(0)=c_{\mathrm{OH}}(0)=n, \phi(0)=$ 0 and $c_{ \pm}(3)=1, c_{\mathrm{H}}(3)=c_{\mathrm{OH}}(3)=n$, and $\phi(3)=-V_{0}$. To improve the numerical stability of the problem, we have made a change of variable, so that the logarithm of the concentration fields have been used as dependent variables instead of the concentration fields themselves.

The code has been successfully validated both against known analytical results in various special cases, and by performing careful mesh-convergence analyses as in Ref. [49]. Subsequently, the model system has been solved for $c_{0}$ increasing from 0.1 to $100 \mathrm{mM}$ in six steps and for $L$ increasing from $1 \mu \mathrm{m}$ to $10 \mathrm{~mm}$ in eight steps. Thus, a total

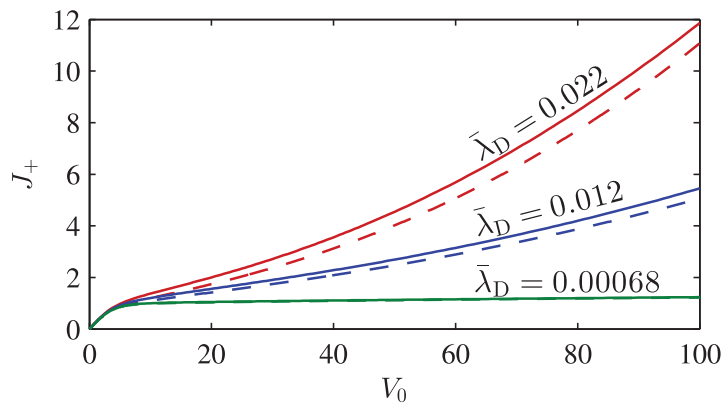

FIG. 6. (Color online) Salt current $J_{+}$plotted versus voltage $V_{0}$ for varying $\bar{\lambda}_{\mathrm{D}}$ neglecting the water-ion current $J_{\mathrm{w}}$. The full lines are numerical simulations and the dashed lines are the corresponding analytical results from Eq. (42) for $\kappa=1$ and with Eqs. (25) and (26) inserted.

of 63 configurations have been investigated. For each set of parameters, the bias voltage $V_{0}$ was varied from 0 to 100 in 160 steps (smaller steps at small $V_{0}$ ). In total, this resulted in 10080 data points of which 8056 have an overlimiting current $J_{+}>1$.

\section{B. Numerical results}

First, we present the results for the case without water ions. In this case, the problem only depends on one parameter, namely, $\bar{\lambda}_{\mathrm{D}}$. In Fig. 6 , the salt current $J_{+}$is plotted versus the bias voltage $V_{0}$ for three values of $\bar{\lambda}_{\mathrm{D}}$ (full lines). It should be noted that the normalization current is different for the three cases. The analytical expression from Eq. (42) with Eqs. (25) and (26) inserted is also shown (dashed lines). For small $\bar{\lambda}_{\mathrm{D}}$, the current saturates at the limiting current as found in the LEN analysis, while significant deviation from the LEN expression is found for larger $\bar{\lambda}_{D}$ values. The seen deviations from the LEN expression agree well with our expectation that in the limit of very large $\bar{\lambda}_{\mathrm{D}}$, a linear $I-V$ curve should result.

The analytical $I-V$ curves are seen to agree well with the numerical results. The main reason for the small discrepancy is that the width of the EDL becomes non-negligible for large $\bar{\lambda}_{\mathrm{D}}$, and therefore the length $L^{*}$ begins to deviate significantly from the assumed value $L^{*}=1$. The wide range of values of $\bar{\lambda}_{\mathrm{D}}$, where the approximation $L^{*}=1$ is valid, is determined in the Supplemental Material, Sec. I A [40].

When water ions are taken into account, the problem depends on the normalized equilibrium constant $n=\sqrt{K_{\mathrm{w}}} / c_{0}$ and the normalized Debye length $\bar{\lambda}_{\mathrm{D}}$. In Fig. 7, the currentvoltage curves are plotted for varying $n$ and for two different values of $\bar{\lambda}_{\mathrm{D}}$. The analytical expression Eq. (42) with Eqs. (25), (26), and (30) inserted is also shown with dashed lines. The light curves shown in the figures are the water-ion currents $J_{\mathrm{w}}$, and it is seen that the salt currents $J_{+}$nearly equal the classical limiting current plus the water-ion current. This is as expected from Eq. (32) since all the considered cases are in the Kharkats limit $\bar{\lambda}_{\mathrm{D}}^{2} / n^{2} \ll 1$. It is seen that several of the curves exhibit the characteristic $S$ shape found in experiments [8,26,46,47]. An interesting observation is that there is a family of curves, an example being the $n=3.2 \times 10^{-4}$ curve in Fig. 7(a), for which the overlimiting current closely resembles the overlimiting 


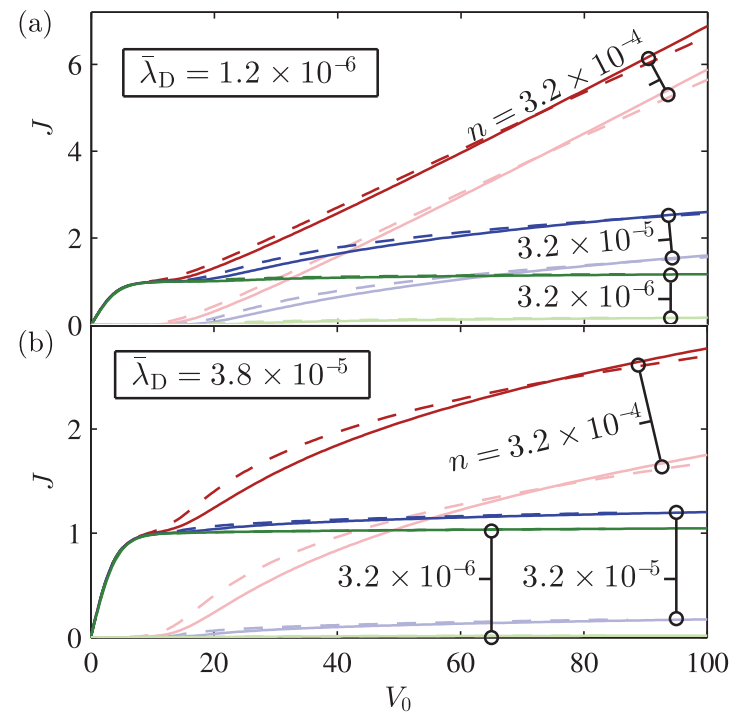

FIG. 7. (Color online) (a) Salt current $J_{+}$(dark) and water-ion current $J_{\mathrm{w}}$ (light) plotted versus voltage $V_{0}$ for $\bar{\lambda}_{\mathrm{D}}=1.2 \times 10^{-6}$ and $n=3.2 \times 10^{-6}, 3.2 \times 10^{-5}$, and $3.2 \times 10^{-4}$. The full lines are numerical simulations and the dashed lines are the corresponding analytical results from Eq. (42) for $\kappa=1$ and with Eqs. (25), (26), and (30) inserted. (b) Same as above, but with $\bar{\lambda}_{\mathrm{D}}=3.8 \times 10^{-5}$.

current caused by EOI $[9,14]$. These curves are, however, found in the $\bar{\lambda}_{\mathrm{D}}^{2} / n^{2} \ll 1$ limit where EOI is suppressed. During a measurement series, where the concentration is varied, one might therefore go from a EOI dominated regime to a water-ion current dominated regime, without observing significant qualitative differences in the $I-V$ curves.

From the analysis in Sec. IV C, it is clear that the overlimiting current may be due to either screening by water ions or the development of an extended space-charge region. Which effect is dominant depends on the parameters of the problem. To illustrate this dependence, the overlimiting current

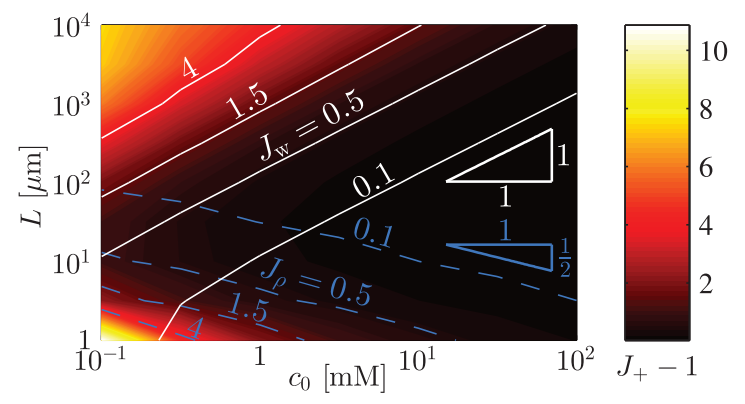

FIG. 8. (Color online) Numerically calculated color plot from 0 (black) to 10.8 (white) of the overlimiting salt current $J_{+}-1=J_{\rho}+$ $J_{\mathrm{w}}$ at $V_{0}=100$ as a function of the reservoir concentration $c_{0}$ and the compartment length $L$. The full lines indicate contours $J_{\mathrm{w}}=0.1$, $0.5,1.5$, and 4.0. The dashed lines are contours for the current due to the extended space-charge region $J_{\rho}=0.1,0.5,1.5$, and 4.0. The slope indications (triangles) show the approximate scalings $L \sim c_{0}^{-1 / 2}$ for the $J_{\rho}$ contours and $L \sim c_{0}$ for the $J_{\mathrm{w}}$ contours from Eqs. (34b) and (34a), respectively. The following parameter values were used in converting from $n$ and $\bar{\lambda}_{\mathrm{D}}$ to $c_{0}$ and $L: \epsilon_{\mathrm{w}}=6.90 \times 10^{-10} \mathrm{~F} / \mathrm{m}$, $V_{\mathrm{T}}=25.8 \mathrm{mV}, e=1.602 \times 10^{-19} \mathrm{C}$, and $K_{\mathrm{w}}=10^{-14} \mathrm{M}^{2}$.
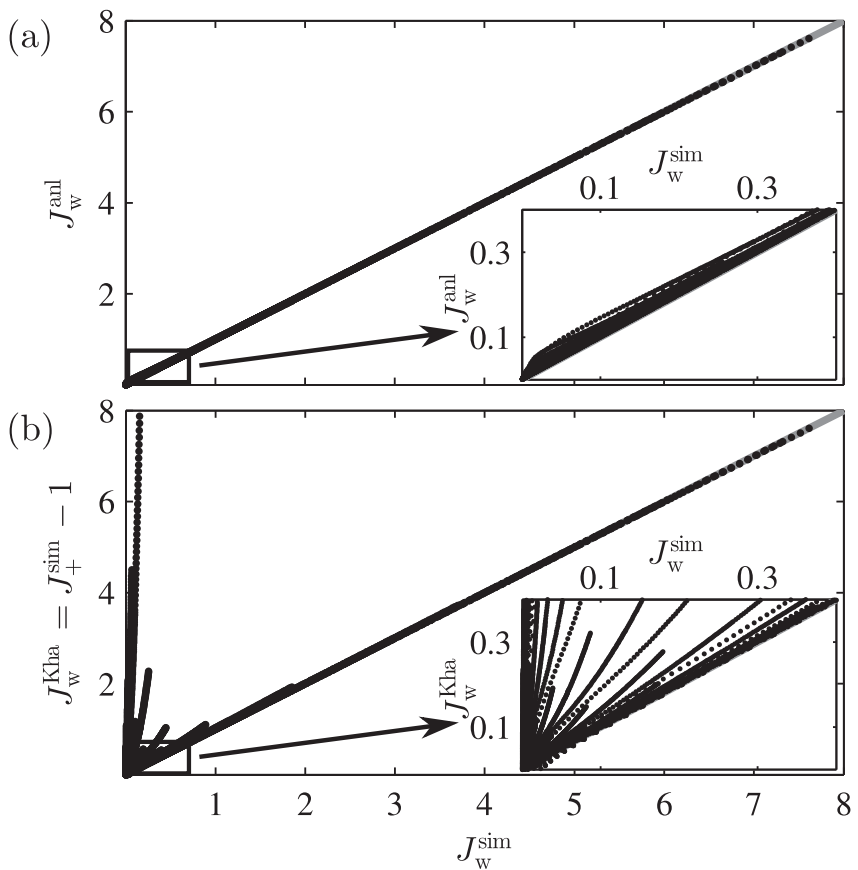

FIG. 9. (a) The analytical water-ion current $J_{\mathrm{w}}^{\mathrm{anl}}$ from Eq. (30) with $\kappa=1$ plotted versus the simulated water-ion current $J_{\mathrm{w}}^{\text {sim }}$ for the 8056 sets of values for $\bar{\lambda}_{\mathrm{D}}, n$, and $J_{+}$, as defined in the last paragraph of Sec. V A, all having an overlimiting current $J_{+}>1$. The inset zooms in on the zero-current limit. (b) Same as above, except that $J_{\mathrm{w}}^{\mathrm{anl}}$ is substituted by the Kharkats expression $J_{\mathrm{w}}^{\text {Kha }}=J_{+}^{\text {sim }}-1$.

at $V_{0}=100$ is plotted in Fig. 8 along with contour lines showing the current due to water-ion screening $J_{\mathrm{w}}$ (white), and charge neutrality violation $J_{\rho}=J_{+}-1-J_{\mathrm{w}}$ (dark).

In the following, we make a more systematic comparison between the analytical model and the results of the numerical simulation. We begin by evaluating the model for water splitting. For each set of parameters $\bar{\lambda}_{\mathrm{D}}, n$, and $J_{+}$used in the simulations the water-ion current $J_{\mathrm{w}}$ was calculated using Eq. (30), and in Fig. 9(a) it is plotted versus the water-ion current which was actually observed in the simulations. Only the cases $J_{+}>1$ are shown since $J_{\mathrm{w}}$ nearly vanishes in the underlimiting regime. It is seen that the developed model captures the majority of the dependence. To better appreciate the level of agreement, the simple Kharkats result $J_{\mathrm{w}}^{\text {Kha }}=$ $J_{+}-1$ is shown in Fig. 9(b).

The total model giving the current-voltage relation for the system has also been evaluated. In Fig. 10, the salt current has been calculated according to Eqs. (25), (26), (30), and (42) and plotted versus the salt current obtained from simulations using the same parameter values. There is seen to be some scatter around perfect agreement between the two models, but the overall behavior is definitely captured by the analytical model.

\section{ADDITION OF ACID OR BASE}

So far, we have investigated systems where the ions derive from a dissolved salt. We will now proceed with a more general treatment, where we allow for some concentration of acid $c_{\mathrm{a}}$ or base $c_{\mathrm{b}}$ in the reservoirs in analogy with Ref. [50]. The acid or 


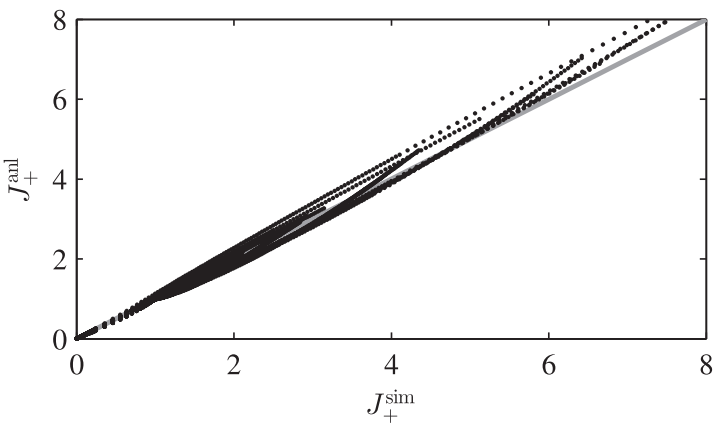

FIG. 10. The analytical salt current $J_{+}^{\text {anl }}$ from Eqs. (25), (26), (30), and (42) with $\kappa=1$ plotted versus the simulated salt current $J_{+}^{\text {sim }}$ for all 1080 sets of values for $\bar{\lambda}_{\mathrm{D}}, n$, and $V_{0}$, as defined in the last paragraph of Sec. V A.

base is assumed to be strong so that it dissociates completely, and for simplicity we assume that the conjugate base to the acid is the same as the negative salt ion and that the conjugate acid to the base is the same as the positive salt ion. For instance, the salt could be $\mathrm{NaCl}$, the acid $\mathrm{HCl}$, and the base $\mathrm{NaOH}$. We use $\kappa=1$ throughout this section.

First, we consider a system where some concentration $c_{\mathrm{b}}$ of base is added to the system. The ion concentrations are normalized with the total cation concentration at the inlet, i.e., the sum of the salt and the base concentrations. We thus have $c_{+}(0)=1, c_{-}(0)=1-c_{\mathrm{b}}$, and $c_{\mathrm{OH}}(0)=c_{\mathrm{b}}$. Like in Sec. IV, hydroxide dominates over hydronium, so the relevant transport equation for the water ions is [Eq. (9b)]

$$
2 J_{\mathrm{w}} \approx \partial_{x} c_{\mathrm{w}}-c_{\mathrm{w}} \partial_{x} \phi,
$$

but with the difference that $c_{\mathrm{w}}(0)=c_{\mathrm{b}}$ rather than $c_{\mathrm{w}}(0)=$ $(1+\delta) n \approx 0$. We can rewrite the transport equation

$$
\begin{aligned}
2 J_{\mathrm{w}} & \approx \partial_{x} c_{\mathrm{w}}-c_{\mathrm{w}} \partial_{x} \phi \\
& =\partial_{x}\left(c_{\mathrm{w}}-c_{\mathrm{b}} e^{\phi}\right)-\left(c_{\mathrm{w}}-c_{\mathrm{b}} e^{\phi}\right) \partial_{x} \phi \\
& =\partial_{x} c_{\mathrm{w}}^{\prime}-c_{\mathrm{w}}^{\prime} \partial_{x} \phi,
\end{aligned}
$$

where $c_{\mathrm{w}}^{\prime} \equiv c_{\mathrm{w}}-c_{\mathrm{b}} e^{\phi}$ and $c_{\mathrm{w}}^{\prime}(0)=0$. The $c_{\mathrm{b}} e^{\phi}$ term behaves exactly like the stationary salt anions, suggesting the introduction of $c_{-}^{\prime} \equiv c_{-}+c_{\mathrm{b}} e^{\phi}$ with $c_{-}^{\prime}(0)=1$.

In conclusion, the present problem can be mapped onto the problem in Sec. IV. Adding a base to a system is therefore equivalent to adding a salt of its conjugate acid. It is noted that to the right of the membrane hydronium dominates the water-ion transport, so in this region it will make a slight difference to add a base to the reservoir.

The situation becomes more complex when an acid is added to the reservoir. In this case, two quite different situations can result, depending on the amount of added acid. For high acid concentrations, the amount of hydronium ions suppress water splitting at the membrane, and the hydronium ions essentially act as a conserved cation. For low acid concentrations, hydroxide may begin to dominate the water-ion transport at some point and water splitting can occur as in the treatment in Sec. IV. In Fig. 11, this situation is illustrated.

To quantify what is meant by "high" and "low" acid concentrations, we analyze the system in more detail. From Fig. 11 it is seen that there are two distinct regions in

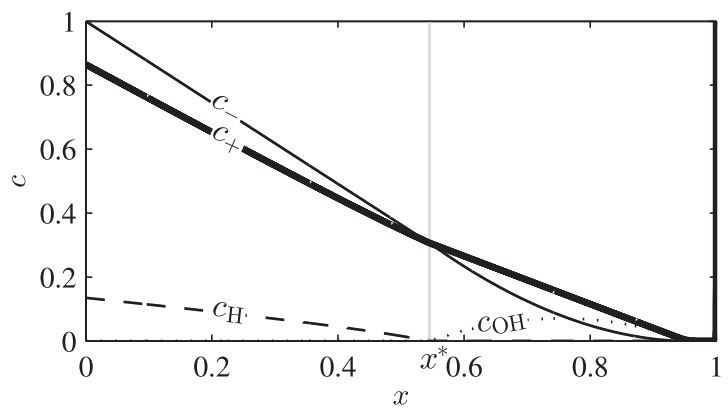

FIG. 11. Numerical simulation of the concentrations of salt ions $\left(c_{+}\right.$and $c_{-}$) and water ions $\left(c_{\mathrm{OH}}\right.$ and $c_{\mathrm{H}}$ ) plotted versus position $x$ in a system with acid concentration $c_{\mathrm{a}}=0.135$ and voltage drop $V_{0}=$ 100. For $x<x^{*}$ (left of the vertical gray line), hydronium behaves as a conserved cation, and the system is well described by a LEN model. For $x>x^{*}$ hydroxide is the dominant water ion, and the system behaves as the aqueous salt solution analyzed in Sec. IV.

the solution. To the left hydronium dominates and there is local electroneutrality, while the right part of the channel is equivalent to the system analyzed in Sec. IV. In the left part of the channel, it is easily found that the concentration fields are given as

$$
\begin{aligned}
& c_{-}=e^{\phi}=1-\left(J_{+}+J_{\mathrm{w}} / \delta\right) x, \\
& c_{\mathrm{H}}=\frac{J_{\mathrm{w}} / \delta}{J_{+}+J_{\mathrm{w}} / \delta} e^{\phi}+\left[c_{\mathrm{a}}-\frac{J_{\mathrm{w}} / \delta}{J_{+}+J_{\mathrm{w}} / \delta}\right] e^{-\phi}, \\
& c_{+}=\frac{J_{+}}{J_{+}+J_{\mathrm{w}} / \delta} e^{\phi}+\left[1-c_{\mathrm{a}}-\frac{J_{+}}{J_{+}+J_{\mathrm{w}} / \delta}\right] e^{-\phi},
\end{aligned}
$$

where the concentration fields are normalized with the sum of the acid and salt concentrations at the inlet and $c_{\mathrm{a}}$ is the normalized acid concentration at the inlet. In the limit where there is no water splitting at the membrane, the currents are just related via the reservoir concentrations of hydronium and salt cation

$$
\frac{J_{\mathrm{w}} / \delta}{J_{+}}=\frac{c_{\mathrm{a}}}{1-c_{\mathrm{a}}}, \text { no water splitting. }
$$

If there is water splitting, there will be a transition point $x^{*}$ where the hydronium concentration vanishes. Solving Eqs. (45a) and (45b) for $x^{*}$ we find

$$
x^{*}=\frac{1}{J_{+}+J_{\mathrm{w}} / \delta}\left[1-\sqrt{1-\frac{J_{+}+J_{\mathrm{w}} / \delta}{J_{\mathrm{w}} / \delta} c_{\mathrm{a}}}\right] .
$$

At that point, the salt concentration is

$$
c^{*} \equiv c_{+}\left(x^{*}\right)=c_{-}\left(x^{*}\right)=\sqrt{1-\frac{J_{+}+J_{\mathrm{w}} / \delta}{J_{\mathrm{w}} / \delta} c_{\mathrm{a}}} .
$$

In the right part of the channel, the electric field is determined by Eq. (14) corrected with the new boundary conditions (47) and (48):

$$
1+\frac{\bar{\lambda}_{\mathrm{D}}^{2}\left(\partial_{x} \phi\right)^{3}}{2\left(J_{+}+J_{\mathrm{w}}\right)}=\left[\frac{J_{+}-J_{\mathrm{w}}}{J_{+}+J_{\mathrm{w}}}\left(x-x^{*}\right)-\frac{c^{*}}{J_{+}+J_{\mathrm{w}}}\right] \partial_{x} \phi .
$$




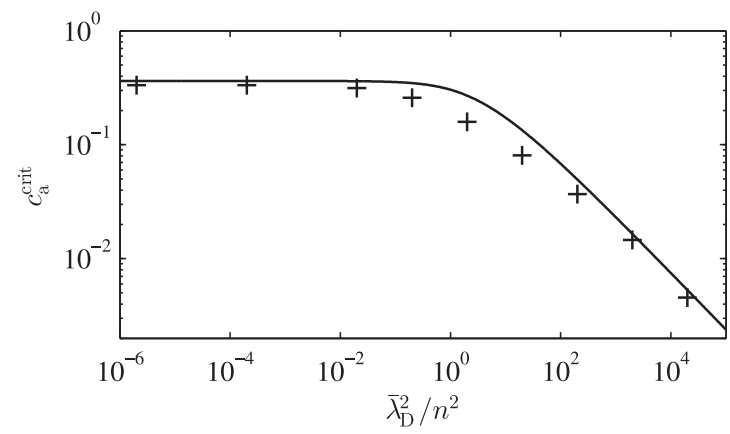

FIG. 12. The critical value $c_{\mathrm{a}}^{\text {crit }}$ of the acid concentration, corresponding to the onset of water-splitting suppression, plotted versus $\bar{\lambda}_{\mathrm{D}}^{2} / n^{2}$. The full line is the analytical expression given in Eq. (52), and the points "+" denote results from numerical simulations.

Inserting the boundary condition (29) and introducing $G \equiv$ $\frac{J_{\mathrm{w}} / \delta}{J_{+}}$this equation can be recast as a quadratic equation for $J_{+}$:

$$
\begin{aligned}
& \frac{\bar{\lambda}_{\mathrm{D}}^{2}}{2}\left(\frac{\sqrt{\delta} G}{n}\right)^{3} J_{+}^{2}-(1-\delta G)\left(\frac{\sqrt{\delta} G}{n}\right) J_{+} \\
& =-\left[\frac{1-\delta G}{1+G}\left(1-c^{*}\right)+c^{*}\right]\left(\frac{\sqrt{\delta} G}{n}\right)+(1+\delta G) .
\end{aligned}
$$

Just at the point where water splitting begins, $G$ will still equal $\frac{c_{\mathrm{a}}}{1-c_{\mathrm{a}}}$ as in Eq. (46) and $c^{*}$ will be very close to 0 . Furthermore, the terms with $\left(\frac{\sqrt{\delta} G}{n}\right)$ dominate over the term $1+\delta G$, so near that point we can simplify the equation as

$$
\frac{\bar{\lambda}_{\mathrm{D}}^{2}}{2}\left(\frac{\sqrt{\delta} G}{n}\right)^{2} J_{+}^{2}-(1-\delta G) J_{+} \approx-\frac{1-\delta G}{1+G} .
$$

This equation has a solution when the determinant is nonnegative, i.e., when

$$
\frac{c_{\mathrm{a}}}{1-c_{\mathrm{a}}}=G \leqslant \frac{1-\delta+\sqrt{(1-\delta)^{2}+4 \delta\left(1+2 \bar{\lambda}_{\mathrm{D}}^{2} / n^{2}\right)}}{2 \delta\left(1+2 \bar{\lambda}_{\mathrm{D}}^{2} / n^{2}\right)} .
$$

For higher values of $c_{\mathrm{a}}$, there are no solutions which allow for water splitting. The value of $c_{\mathrm{a}}$ for which there is an equal sign in Eq. (52), corresponding to the onset of water-splitting suppression, is denoted the critical acid concentration $c_{\mathrm{a}}^{\text {crit }}$. In Fig. 12, analytical and numerical results for the critical acid concentration are plotted versus $\bar{\lambda}_{\mathrm{D}}^{2} / n^{2}$. Numerically, the critical concentration is determined as follows. When there is no water splitting, the currents are related as in Eq. (46). The critical concentration is then defined to be the minimum value of $c_{\mathrm{a}}$ for which $\frac{J_{\mathrm{w}} / \delta}{J_{+}} \geqslant 1.01 \frac{c_{\mathrm{a}}}{1-c_{\mathrm{a}}}$, within the voltage sweep interval $0<V_{0}<100$.

The existence of a critical acid concentration, and its approximate value, is expected to be a robust prediction, which is valid even under circumstances where the assumption of an equilibrated water-dissociation reaction breaks down.

\section{GENERALIZATION TO OTHER SYSTEMS}

The analysis in this paper has so far been concerned with the reservoir-compartment-membrane-compartment-reservoir system sketched in Fig. 1. The difficult part of the analysis, carried out in Sec. IV A, is however largely system independent, and in this section we show examples of generalizations to other systems.

In Sec. IV A, we find the potential drop over the inlet compartment as a function of the currents. The only other parameters we use in the analysis are the reservoir salt concentration $c_{+}(0)=c_{-}(0)=1$ and water-ion concentration $c_{\mathrm{H}}(0)=c_{\mathrm{OH}}(0)=n \approx 0$. By modifying these boundary conditions appropriately, the results for the already studied system can be related to other systems exhibiting concentration polarization. We give two such examples in the following.

\section{A. Membrane-compartment-membrane system}

We consider a system composed of a compartment of normalized length unity with cation-selective membranes on either side. In the compartment is an electrolyte of normalized concentration unity.

Whereas the membranes in an electrodialysis cell alternate between cation and anion selectivity [1], both membranes in this system have the same selectivity. We can thus regard this system as the symmetrical counterpart to the asymmetric electrodialysis cell. With respect to the symmetry, the system has similarities to those studied in Refs. [39,45,51], except for the fact that they have electrodes as ion-selective elements rather than membranes.

Due to the ion-selective membranes, the anions can not leave the compartment, and we have the constraint

$$
\int_{0}^{1} c_{-} d x=1
$$

instead of the boundary condition $c_{-}(0)=1$ from before. As in the original analysis, we only consider the part of the system lying outside the quasiequilibrium EDL's adjoining the membranes. Since hydronium dominates inside the membranes and hydroxide dominates in the compartment, there is a crossover approximately at $x=0$ where the concentrations are equal $c_{\mathrm{H}}(0)=c_{\mathrm{OH}}(0)=n \approx 0$ as in the earlier analysis. Our task is now to find a value of $\kappa$ which is consistent with the constraint in Eq. (53). We use that the anion concentration is negligible outside the LEN region, and to a good approximation we therefore have

$$
\int_{0}^{1} c_{-} d x=\int_{0}^{1} c_{-}^{\mathrm{LEN}} d x
$$

Inserting from Eq. (35b) we obtain

$$
\begin{aligned}
& \int_{0}^{1} c_{-}^{\mathrm{LEN}} d x \\
& =\int_{0}^{\min \left\{1, \kappa /\left(J_{+}-J_{\mathrm{w}}\right)\right\}}\left[\kappa-\left(J_{+}-J_{\mathrm{w}}\right) x\right]^{j} d x \\
& = \begin{cases}\frac{\kappa^{j+1}}{2 J_{+}} & \text {for } \kappa \leqslant\left(J_{+}-J_{\mathrm{w}}\right), \\
\frac{\kappa^{j+1}}{2 J_{+}}-\frac{\left[\kappa-\left(J_{+}-J_{\mathrm{w}}\right)\right]^{j+1}}{2 J_{+}} & \text {for } \kappa \geqslant\left(J_{+}-J_{\mathrm{w}}\right) .\end{cases}
\end{aligned}
$$


The lower case corresponds to the underlimiting case where water splitting is negligible. In that case, we can simplify as

$$
\int_{0}^{1} c_{-}^{\mathrm{LEN}} d x \approx \kappa-\frac{J_{+}}{2} \text { for } \kappa \geqslant\left(J_{+}-J_{\mathrm{w}}\right) .
$$

From the constraint we then find

$$
\kappa= \begin{cases}\left(2 J_{+}\right)^{\frac{J_{+}-J_{\mathrm{W}}}{2 J_{+}}} & \text {for } 2 \lesssim J_{+}, \\ 1+\frac{J_{+}}{2} & \text { for } 2 \gtrsim J_{+} .\end{cases}
$$

With this expression for $\kappa$ the boundary condition (29) yields a transcendental equation for the relation between $J_{+}$and $J_{\mathrm{w}}$. Using this relation together with Eq. (25), the $I-V$ curve for the system can be found. In the case of negligible water-ion current, the $I-V$ curve can be obtained directly by inserting Eq. (57) in Eq. (25).

\section{B. Electrode-compartment-electrode system}

We consider a system composed of a compartment of normalized length unity with electrodes on either side. In the compartment is an electrolyte of normalized concentration unity. The electrolyte is formed by dissolving a salt of the electrode metal, which in the case of copper electrodes could be $\mathrm{CuSO}_{4}$. Since only the metal ions can enter or leave the electrodes, the electrodes act as ion-selective elements similarly to the previously studied membranes. This system is similar to those studied in Refs. [39,45,51]. However, we do not consider the potential drops in the EDL's adjoining the electrodes. As before, the anions (here $\mathrm{SO}_{4}^{-}$) can not leave the system so we have the constraint

$$
\int_{0}^{1} c_{-} d x=1
$$

So far, the system is equivalent to the membrane-compartmentmembrane system. However, whereas the membranes are only sensitive to the charge of the ions, the electrodes also select based on the ion species. This implies that the hydronium ions can not leave the system either, and they will accumulate in the left part of the compartment while hydroxide accumulates to the right. We can put this in terms of a constraint by noting that hydronium and hydroxide are created in equal amounts

$$
\int_{0}^{1} c_{\mathrm{H}} d x=\int_{0}^{1} c_{\mathrm{OH}} d x
$$

In Sec. VI, we treated a system where hydronium dominates to the left due to the addition of an amount of acid $c_{\mathrm{a}}$ to the reservoir. By letting $c_{\mathrm{a}}$ vary to satisfy Eq. (59) and letting $\kappa$ vary to satisfy Eq. (58), we can therefore map the present problem onto the original reservoir-compartment-membranecompartment-reservoir problem. Because of the need to split the system into a hydronium dominated part and a hydroxide dominated part, this mapping is however significantly more involved than the one in Sec. VII A. For this reason, we will not bring the full analysis here, but we note that it is in principle a simple matter which mainly requires some bookkeeping with the different regions.

\section{DISCUSSION}

The results presented in this paper are based on the assumption of a locally equilibrated water-dissociation reaction. Whether this assumption is correct is at present not known, but since our theoretical predictions rely on this assumption, an experimental test of our predictions would constitute a (partial) test of the underlying assumptions.

From the analytical model, several useful results are obtained. Our main theoretical result Eq. (25) provides the potential $\phi(1)$ at the beginning of the EDL, for a general ion-selective interface with both a water-ion current and the extended space-charge region taken into account. In certain limits, this result can be simplified to Eq. (34). The effects of water splitting are accounted for by Eq. (30), which provides a relation between the salt current $J_{+}$and the water-ion current $J_{\mathrm{w}}$.

The potential drop across the EDL and the rest of the system depends on the specific ion-selective interface and gives a small correction to the potential. For the specific ion-selective membrane system studied in this work, these corrections are included in Eq. (42), and in Sec. VII we show examples of generalizations to other systems. The model also provides the detailed structure of the extended space-charge region and yields the simple expression Eq. (20) for the maximum value of the charge density $\rho_{\mathrm{el}}$. The analytical model has been successfully tested against direct numerical simulations (see, e.g., Fig. 10 containing a plot of $J_{+}^{\text {anl }}$ versus $J_{+}^{\text {sim }}$ ).

Even if the fundamental assumption of a locally equilibrated water-dissociation reaction is not entirely correct, the analytical model is still useful since it provides an upper bound to the water-ion current, as long as the equilibrium constant $K_{\mathrm{w}}$ does not change appreciably. For instance, Fig. 8 shows that in a large portion of the parameter space the influence of water ions is negligible. Since this is an upper bound, we can conclude that water splitting is unimportant for these parameter values regardless of the reaction speed. As described in Sec. IV C, it would be a relatively simple matter to extend the analysis to allow for a varying $K_{\mathrm{w}}$.

A strength of the analysis given in this paper is that several of the derived expressions are comparatively easy to test experimentally since they only depend on a few parameters which can either be estimated or fitted. Consider, for instance, Eq. (31) for the water-ion current $J_{\mathrm{w}}$, which in dimensionful terms can be rewritten as $\tilde{J}_{\mathrm{w}}$ :

$$
\tilde{J}_{\mathrm{w}} \approx \frac{2 D_{\mathrm{OH}}}{\gamma D_{+}}\left[-1+\sqrt{1+\gamma\left(\frac{\tilde{J}_{+}}{J_{\mathrm{lim}}}-1\right)}\right] J_{\mathrm{lim}},
$$

where $J_{\lim }=2 D_{+} c_{0} / L$ is the limiting current, and where $\gamma=$ $\left(D_{\mathrm{OH}} / D_{\mathrm{H}}\right) c_{0} \epsilon_{\mathrm{w}} k_{\mathrm{B}} T /\left(L^{2} K_{\mathrm{w}} e^{2}\right)$ is a dimensionless parameter. Given knowledge of the reservoir concentration $c_{0}$ and the length $L$ of the diffusive boundary layer, it is possible to calculate $\gamma$ and $J_{\text {lim }}$ from the definitions. Since Eq. (60) is derived under the assumption of an equilibrated waterdissociation reaction, a set of experimental data which fits it would corroborate that assumption and our model.

Another prediction which can be experimentally tested is the existence of a critical acid concentration $c_{\mathrm{a}}^{\text {crit }}$ for the onset of water-splitting suppression, which may be tested 
experimentally using the titration method [26,27]. For acid concentrations $c_{\mathrm{a}}$ above $c_{\mathrm{a}}^{\text {crit }}$, we predict that the water-ion current and the salt current will be proportional. When $c_{\mathrm{a}}$ is reduced below $c_{\mathrm{a}}^{\text {crit }}$, given by Eq. (52), the water-ion current will begin to exceed the value given by Eq. (46). If, instead, a base is added to the system, we predict that there will be no such critical concentration, and adding an amount of base will in fact be equivalent to adding the same amount of salt. It should be noted that these predictions assume that an added acid or base does not significantly alter the properties of the membrane through chemical reactions. For a chemically stable membrane such as nafion, this should be a good assumption.

In the analytical treatment, it was found that water splitting will act to suppress EOI in the limit of $\bar{\lambda}_{\mathrm{D}}^{2} / n^{2} \ll 1$. We have not verified this prediction by full $3 \mathrm{D}$ numerical simulations of EOI, but since water splitting begins at a lower voltage than EOI, it is likely that a suppression of EOI will in fact occur.

Lastly, we emphasize the simplicity and versatility of the employed mathematical method. The reduction of the problem to the simple algebraic equation (17) for the electric field hugely simplifies the analysis and gives a good description of the ESC not involving singularities: unlike in the method of matched asymptotic expansions, the fields in this approach do not diverge at the entrance to the $\mathrm{ESC}$, and for this reason closed-form expressions for every relevant quantity can be obtained with ease.

\section{CONCLUSION}

In this paper, we have developed analytical and numerical models for the current through and the voltage drop across an ion-selective interface, taking into account both the effect of the extended space-charge region adjoining the interface as well as the effect of water splitting and screening by water ions. Specifically, we have investigated the transport through an ion-selective membrane, but the fundamental results apply to any ion-selective interface.

The fundamental assumption in the analysis is that the autodissociation of water happens on a much shorter time scale than the transport of water ions, i.e., we study transport-limited processes. The validity of this assumption is dependent on the particular system under study, but in general the model gives an upper bound to the currents which can be obtained, given a fixed equilibrium constant $K_{\mathrm{w}}$, for the water-splitting reaction.

In the analytical model, the assumption of quasiuniform charge density distribution has been used to simplify the treatment. The analytical and the numerical models compare favorably and both models exhibit some of the characteristic behavior observed in experiments. The developed analytical model is readily testable in experiments, as it gives both detailed expressions for the current-voltage characteristics, simple scaling laws with few parameters, and predictions about the system behavior upon addition of an acid or a base.
[1] V. V. Nikonenko, N. D. Pismenskaya, E. I. Belova, P. Sistat, P. Huguet, G. Pourcelly, and C. Larchet, Adv. Colloid Interface Sci. 160, 101 (2010).

[2] S. J. Kim, S. H. Ko, K. H. Kang, and J. Han, Nat. Nanotechnol. 5, 297 (2010).

[3] M. Park, X. Zhang, M. Chung, G. B. Less, and A. M. Sastry, J. Power Sources 195, 7904 (2010).

[4] M. Winter and R. J. Brodd, Chem. Rev. 104, 4245 (2004).

[5] P. Malek, J. M. Ortiz, B. S. Richards, and A. I. Schaefer, J. Membr. Sci. 435, 99 (2013).

[6] Y. Tanaka, H. Uchino, and M. Murakami, Membr. Water Treat. 3, 63 (2012).

[7] V. Etacheri, R. Marom, R. Elazari, G. Salitra, and D. Aurbach, Energy Environ. Sci. 4, 3243 (2011).

[8] M. Taky, G. Pourcelly, F. Lebon, and C. Gavach, J. Electroanal. Chem. 336, 171 (1992).

[9] F. Maletzki, H. W. Rösler, and E. Staude, J. Membr. Sci. 71, 105 (1992).

[10] W. H. Smyrl and J. Newman, Trans. Faraday Soc. 63, 207 (1967).

[11] I. Rubinstein and L. Shtilman, J. Chem. Soc., Faraday Trans. 2 75, 231 (1979).

[12] E. Yariv, Phys. Rev. E 80, 051201 (2009).

[13] I. Rubinstein and B. Zaltzman, Phys. Rev. E 62, 2238 (2000).

[14] I. Rubinshtein, B. Zaltzman, J. Pretz, and C. Linder, Russ. J. Electrochem. 38, 853 (2002).

[15] I. Rubinstein, J. Phys. Chem. 81, 1431 (1977).

[16] Y. I. Kharkats, J. Electroanal Chem. 105, 97 (1979).

[17] M. B. Andersen, M. van Soestbergen, A. Mani, H. Bruus, P. M. Biesheuvel, and M. Z. Bazant, Phys. Rev. Lett. 109, 108301 (2012).
[18] E. V. Dydek, B. Zaltzman, I. Rubinstein, D. S. Deng, A. Mani, and M. Z. Bazant, Phys. Rev. Lett. 107, 118301 (2011).

[19] S. H. Behrens and D. G. Grier, J. Chem. Phys. 115, 6716 (2001).

[20] W. B. S. de Lint, P. M. Biesheuvel, and H. Verweij, J. Colloid Interface Sci. 251, 131 (2002).

[21] K. L. Jensen, J. T. Kristensen, A. M. Crumrine, M. B. Andersen, H. Bruus, and S. Pennathur, Phys. Rev. E 83, 056307 (2011).

[22] M. B. Andersen, J. Frey, S. Pennathur, and H. Bruus, J. Colloid Interface Sci. 353, 301 (2011).

[23] M. Block and J. A. Kitchener, J. Electrochem. Soc. 113, 947 (1966).

[24] A. A. Sonin and G. Grossman, J. Phys. Chem. 76, 3996 (1972).

[25] R. Simons, Electrochim. Acta 30, 275 (1985).

[26] V. V. Nikonenko, N. D. Pismenskaya, and E. I. Volodina, Russ. J. Electrochem. 41, 1205 (2005).

[27] V. I. Zabolotsky, V. V. Nikonenko, N. D. Pismenskaya, E. V. Laktionov, M. K. Urtenov, H. Strathmann, M. Wessling, and G. H. Koops, Sep. Purif. Technol. 14, 255 (1998).

[28] Y. Tanaka and M. Seno, J. Chem. Soc., Faraday Trans. 182 , 2065 (1986).

[29] V. Mavrov, W. Pusch, O. Kominek, and S. Wheelwright, Desalination 91, 225 (1993).

[30] Y. Tanaka, J. Membr. Sci. 350, 347 (2010).

[31] R. Simons, Desalination 28, 41 (1979).

[32] L. Jialin, W. Yazhen, Y. Changying, L. Guangdou, and S. Hong, J. Membr. Sci. 147, 247 (1998).

[33] R. Simons, Electrochim. Acta 29, 151 (1984).

[34] C.-O. Danielsson, A. Dahlkild, A. Velin, and M. Behm, Electrochim. Acta 54, 2983 (2009). 
[35] V. I. Zabolotskii, V. V. Nikonenko, N. M. Korzhenko, R. R. Seidov, and M. K. Urtenov, Russ. J. Electrochem. 38, 810 (2002).

[36] C. Heitner-Wirguin, J. Membr. Sci. 120, 1 (1996).

[37] T. Xu, J. Membr. Sci. 263, 1 (2005).

[38] R. Nagarale, G. Gohil, and V. K. Shahi, Adv. Colloid Interface Sci. 119, 97 (2006).

[39] K. T. Chu and M. Z. Bazant, SIAM J. Appl. Math. 65, 1485 (2005).

[40] See Supplemental Material at http://link.aps.org/supplemental/ 10.1103/PhysRevE.89.042405 for an assessment of the impact of each approximation and the limits of its applicability.

[41] M. Urtenov, E. V. Kirillova, N. M. Seidova, and V. V. Nikonenko, J. Phys. Chem. B 111, 14208 (2007).

[42] J. Newman, Trans. Faraday Soc. 61, 2229 (1965).
[43] L. Højgaard Olesen, M. Z. Bazant, and H. Bruus, Phys. Rev. E 82, 011501 (2010).

[44] A. S. Khair, Phys. Fluids 23, 072003 (2011).

[45] M. Z. Bazant, K. T. Chu, and B. J. Bayly, SIAM J. Appl. Math. 65, 1463 (2005).

[46] J. J. Krol, M. Wessling, and H. Strathmann, J. Membr. Sci. 162, 145 (1999).

[47] M. Taky, G. Pourcelly, and C. Gavach, J. Electroanal. Chem. 336, 195 (1992).

[48] C. L. Druzgalski, M. B. Andersen, and A. Mani, Phys. Fluids 25, 110804 (2013).

[49] M. M. Gregersen, M. B. Andersen, G. Soni, C. Meinhart, and H. Bruus, Phys. Rev. E 79, 066316 (2009).

[50] Y. I. Kharkats and A. V. Sokirko, J. Electroanal. Chem. 303, 27 (1991).

[51] E. Yariv, SIAM J. Appl. Math. 71, 2131 (2011). 